\title{
Double-exchange mechanisms for Mn-doped III-V ferromagnetic semiconductors
}

\author{
P. M. Krstajić and F. M. Peeters \\ Departement Natuurkunde, Universiteit Antwerpen (Campus Drie Eiken), Universiteitsplein 1, B-2610 Antwerpen, Belgium \\ V. A. Ivanov \\ N.S. Kurnakov Institute of the General and Inorganic Chemistry of the Russian Academy of Sciences, Leninskii Prospect 31, \\ 117907 Moscow, Russia \\ V. Fleurov* \\ Raymond and Beverly Sackler Faculty of Exact Sciences, School of Physics and Astronomy, Tel Aviv University, Ramat Aviv, \\ 69978 Tel Aviv, Israel \\ K. Kikoin \\ Physics Department, Ben-Gurion University, 84105 Beer-Sheva, Israel \\ (Received 23 November 2003; revised manuscript received 23 June 2004; published 22 November 2004)
}

\begin{abstract}
A microscopic model of indirect exchange interaction between transition metal impurities in dilute magnetic semiconductors (DMS) is proposed. The hybridization of the impurity $d$-electrons with the heavy hole band states is mainly responsible for the exchange of electrons between the impurities, whereas the Hund rule for the electron occupation of the impurity $d$-shells makes it spin selective. The model is applied to such systems as $n$-type $(\mathrm{Ga}, \mathrm{Mn}) \mathrm{N}$ and $p$-type $(\mathrm{Ga}, \mathrm{Mn}) \mathrm{As}$, $p$-type $(\mathrm{Ga}, \mathrm{Mn}) \mathrm{P}$. In $n$-type DMS with $\mathrm{Mn}^{2+/ 3+}$ impurities the exchange mechanism is rather close to the kinematic exchange proposed by Zener for mixed-valence Mn ions. In $p$-type DMS ferromagnetism is governed by the kinematic mechanism involving the kinetic energy gain of the heavy hole carriers caused by their hybridization with $3 d$ electrons of $\mathrm{Mn}^{2+}$ impurities. Using the molecular field approximation, the Curie temperatures $T_{C}$ are calculated for several systems as functions of the impurity and hole concentrations. Comparison with the available experimental data shows a good agreement.
\end{abstract}

DOI: 10.1103/PhysRevB.70.195215 PACS number(s): 71.20.Nr, 71.55.Eq, 75.50.Pp, 77.80.Bh

\section{INTRODUCTION}

Dilute magnetic semiconductors (DMS) are semiconductors, in which transition or rareearth metal atoms randomly replace a fraction of atoms in one sublattice. Transition metal (TM) impurities can easily enter the host semiconductor, due to their high abundance and diffusivity. In the group IV elemental semiconductors, they occupy mainly interstitial positions, whereas in III-V semiconductors the $3 d \mathrm{TM}$ impurities usually substitute group III atoms. The metastability of the zinc blende phase of the DMS (III,Mn)V compounds and low solubility of manganese in these materials were the major obstacles for a synthesis of dilute magnetic semiconductors. ${ }^{1}$ However, the idea to combine in DMS the charge degrees of freedom of hole or electron carriers with the spin degrees of freedom of magnetic impurities became reality after a new doping technique based on nonequilibrium low temperature molecular beam epitaxy (LT-MBE) (Refs. 2 and 3) was developed. The discovery ${ }^{4}$ of ferromagnetic (FM) ordering in Mn doped InAs with the $T_{C}=7.5 \mathrm{~K}$ Curie temperature fueled DMS studies that resulted in fabrication of $(\mathrm{Ga}, \mathrm{Mn}) \mathrm{As}$ compounds with $T_{C}=110 \mathrm{~K}$ (Refs. 2 and 5) or even $T_{C}=140 \mathrm{~K} .{ }^{6}$ More recently ${ }^{7-10}$ Curie temperatures exceeding $150 \mathrm{~K}$ were reported in $(\mathrm{Ga}, \mathrm{Mn}) \mathrm{As}$. An above room temperature ferromagnetism was announced ${ }^{11,12}$ also in $\mathrm{GaN}$ and $p$-type GaP doped with $\mathrm{Mn}$. Advanced III-V growth techniques such as metal organic vapor phase epitaxy (MOVPE), or metal organic chemical vapor deposition (MOCVD) together with LT-MBE can produce good quality
DMS with various element combinations. A unique coexistence of high-temperature magnetism and semiconductor properties opens new venues of fundamental studies and applications of DMS's.

Although FM order in (Ga,Mn)As with $T_{C}=60 \mathrm{~K}$ has been observed for the first time as early as in $1996,{ }^{13}$ its microscopic origin still remains not well understood. Extrapolating from $(\mathrm{Ga}, \mathrm{Mn}) \mathrm{As}, \mathrm{FM}$ ordering with high $T_{C}$ was predicted for $p$-type (Ga,Mn)N (Ref. 14) and found in Ref. 12. However, a direct extrapolation of the trends known for the GaAs-based materials, with relatively narrow forbidden energy gap to the wide gap GaN DMS, is not well founded. The differences in formation of the FM order arise from the differences in the structure of chemical bonds between the Mn impurity and valence electrons in various III-V host semiconductors. Our purpose is to pinpoint these differences and to construct a microscopic theory of the double exchange in dilute magnetic semiconductors.

Three venues may be pursued to study magnetic states in DMS's. According to the first approach, an effective spin Hamiltonian is chosen and the corresponding exchange coupling constant is calculated in the course of this derivation. The major part of the available descriptions of the FM order in such DMS's as (Ga,Mn)As, (Ga,Mn)P, and (Ga,Mn)N, are based on semiphenomenological models, postulating the existence of local magnetic moments on the Mn sites, as well as an indirect exchange between these moments and the electrons in the valence band of the host. ${ }^{15-18}$ Sometimes the role of shallow acceptor levels, existing in the first two of these 
systems,${ }^{19}$ or of resonance levels is emphasized. ${ }^{20}$ Impurities interacting with the free carriers due to an effective RRKY interaction were discussed in Ref. 21. That paper considered also a Hubbard-type model for magnetic impurities in DMS, which made it closer to the third approach, to be described below.

The second approach is based on an extended cluster method, where a cubic supercell with a magnetic Mn ion in its center is used to calculate the density of spin polarized states of a "homogeneously doped" crystal (see e.g., Refs. 22 and 23). The resulting picture provides information about positions and occupations of Mn-related majority and minority "bands." Sometimes an effective $s-d$ exchange Hamiltonian is introduced with the effective exchange constant fitting the numerical data (see Ref. 22 and the first paper in Ref. 23). Local spin density approximation in combination with coherent potential approach (CPA) describing averaged properties of alloys was used in papers ${ }^{24}$ to analyze the onset of FM order in DMS systems. Several important parallels between these calculations and our results will be discussed below.

According to the third approach, realistic exchange parameters should be derived within a microscopic theory based on the knowledge of the electronic structure of III-V semiconductors doped with TM impurities. An exhaustive microscopic theory for isolated TM impurities in semiconductors was constructed more than two decades ago starting with the papers of Refs. 25 and 26 (see the monographs Ref. 27 for a detailed description and Ref. 28 where a powerful numeric approach has been developed). This theory contains all the ingredients necessary for an accurate derivation of the indirect magnetic exchange between impurities. Our first attempt ${ }^{29}$ to apply this theory to $(\mathrm{Ga}, \mathrm{Mn}) \mathrm{As}$ resulted in a quantitative description of the hole concentration dependent Curie temperature $T_{C}$ in $p$-type DMS's within the framework of a very simple model Hamiltonian, in which the effective kinematic exchange interaction arises from an exchange of electrons between the occupied $d$-states of the half-filled $d$-shell of the Mn impurity via empty heavy hole $(h h)$ states near the top of the valence band.

Here we present a general theory of the double exchange interaction in Mn-doped III-V semiconductors, which covers both $p$ - and $n$-type materials. The theory establishes differences between the exchange mechanisms in these two cases. We show that the Zener mechanism ${ }^{30}$ in its classical form is realized in $n$-type $(\mathrm{Ga}, \mathrm{Mn}) \mathrm{N}$, whereas the $p$-type systems $(\mathrm{Ga}, \mathrm{Mn}) \mathrm{As}$, $(\mathrm{Ga}, \mathrm{Mn}) \mathrm{P}$ and, possibly, $p-(\mathrm{Ga}, \mathrm{Mn}) \mathrm{N}$ are examples of another type of indirect (kinematic) exchange interaction, which was not observed in the family of Mn oxides described by the Zener theory of FM insulators. Besides, we discuss similarities and differences between the description based on Zener double exchange ${ }^{30}$ and Vonsovskii-Zener $d p$-exchange ${ }^{31}$ accepted in many phenomenological theories. ${ }^{15-18}$ While developing further the microscopical theory ${ }^{29}$ we substantiate the applicability of orbitally nondegenerate two-site Anderson model ${ }^{32,33}$ to the case of $\mathrm{Mn}^{3+}$ impurities and include explicitly the contribution of impurity band in the double interimpurity exchange.

The paper is organized as follows: Sec. II presents a description of the microscopic model including the calculation of the exchange interaction between TM impurities. Since this calculation appears to be rather involved, its mathematical details are given in the Appendix. In Sec. III we apply the results obtained in Sec. II to $p$-type semiconductors, find the kinematic exchange interaction and use the molecular field approximation to calculate the Curie temperature as a function of the Mn content and hole concentration for $p$-type $(\mathrm{Ga}, \mathrm{Mn}) \mathrm{As}$ and $(\mathrm{Ga}, \mathrm{Mn}) \mathrm{P}$. The results are compared with the available experimental data. The $n$-type $(\mathrm{Ga}, \mathrm{Mn}) \mathrm{N}$ DMS, which possesses specific features differing it from other DMS's, is discussed in Sec. IV. Finally, the scope of the theory and comparison with other approaches are summarized in the concluding Sec. V.

\section{INDIRECT EXCHANGE INTERACTION BETWEEN TWO MAGNETIC IMPURITIES}

\section{A. Bands and bonds in Mn-doped III-V compounds}

As is shown in Refs. 27 and 28, the electronic spectrum of isolated substitutional TM in III-V semiconductors is predetermined by the structure of chemical bonds (hybridization) between the $3 d$-orbitals of impurity ions and the $p$-orbitals of the valence band electrons, whereas their magnetic state is governed by the Coulomb and exchange interactions within the $3 d$-atomic shell modified by hybridization with the electron states of the crystalline environment. According to Refs. 34-38, the localized TM $d$-states possess either $t_{2}$ or $e$ symmetry of the crystal point symmetry group $T_{d}$. The $e$ states are practically nonbonding $d$-states, whereas the $t_{2}$ states form bonding and antibonding configurations with the $p$-states of the valence band. The two latter types of states are classified as the crystal field resonances (CFR), in which the $d$-component dominates, and the dangling bond hybrids (DBH) with predominantly $p$-type contribution of the valence band states. It is crucially important that the absolute positions of CFR levels relatively weakly depend on the band structure of the host semiconductor material. The CFR levels are pinned mainly to the vacuum energy, and this pinning is modulated by the counterbalanced interactions with valence and conduction band states. ${ }^{27,39}$ The DBH states are more intimately connected with the peak in the density of states of the heavy hole band, which dominates the $p d$-hybridization.

The variety of electronic and magnetic properties of $\mathrm{Mn}$ and other TM ions in different III-V host semiconductors stems from these universal chemical trends. Among them, the Mn impurity exhibits peculiar features. According to its position in the series of the TM elements, a neutral $\mathrm{Mn}^{3+}$ on a Ga-site is expected to have a $3 d^{4}$ configuration. However, the $\mathrm{Mn}$ ion retains the fifth electron in its $3 d$ shell, because of an exceptional stability of the high-spin half-filled $3 d^{5}$ state resulting from the strong intra-atomic Hund interaction. The Mn impurities not only introduce magnetic moments in the p-type (III,Mn)V compounds but also create potentials attracting holes, i.e., act as acceptors. Therefore, the neutral substitution impurity state is $A^{0}\left(3 d^{5} \bar{p}\right)$, where $\bar{p}$ stands for the loosely bound hole, and the manganese provides both holes and magnetic moments to the host. Here $A$ is the spectroscopic notation indicating the irreducible representation of 
the state (more about these notations can be found, e.g., in Ref. 27).

Existence of the complex $\mathrm{Mn} d^{5} \bar{p}$ in lightly doped bulk GaAs is detected by electron spin resonance ${ }^{40}$ and infrared spectroscopy. ${ }^{41}$ An acceptor level is found inside the energy gap at $110 \mathrm{meV}$ above the top of the valence band. This value is much higher than $30 \mathrm{meV}$, resulting from conventional effective mass theory, ${ }^{40}$ meaning that the treatment of the Mn impurities in III-V compounds requires a more refined approach. On the other hand, the presence of negatively ionized complexes $A^{-}\left(3 d^{5}\right)$ is detected in $(\mathrm{Ga}, \mathrm{Mn})$ As epilayers grown by low temperature molecular beam epitaxy, ${ }^{42}$ which indicates that both mobile and localized holes exist in FM (Ga,Mn)As. The coexistence of weakly and strongly localized states is also in accordance with ac conductivity experiments. ${ }^{43}$

Apparently, a similar impurity electronic structure is realized in $(\mathrm{Ga}, \mathrm{Mn}) \mathrm{P}$. But in contrast, $\mathrm{Mn}$ in the wide gap $\mathrm{GaN}$ semiconductor releases its fifth $d$-electrons to the valence band similarly to all other TM impurities, and remains in a $\mathrm{Mn}^{3+}\left(d^{4}\right)$ state, unless the sample is deliberately $n$-doped. This state is neutral and there is no need to bind a hole in order to maintain the neutrality of the ground state. This leaves no room for a direct extrapolation from $(\mathrm{Ga}, \mathrm{Mn})$ As to $(\mathrm{Ga}, \mathrm{Mn}) \mathrm{N}$, and the kinematic inter-impurity exchange mechanisms in these two systems should be considered separately.

Due to a large mismatch between positions of the valence bands in $\mathrm{GaAs}, \mathrm{GaP}$ on one side and in $\mathrm{GaN}$ on the other side, the $\operatorname{Mn}\left(d^{5} / d^{4}\right)$ CFR level falls deep in the valence band in GaAs, GaP, but it appears within the forbidden energy gap in GaN. The CFR nature of $\operatorname{Mn}\left(d^{5} / d^{4}\right) t_{2}$-level near the middle of the gap for $(\mathrm{Ga}, \mathrm{Mn}) \mathrm{N}$ is confirmed experimentally ${ }^{44}$ and follows also from numerical calculations. ${ }^{23}$ This $t_{2}$-level is empty in the neutral state $\mathrm{Mn}^{3+}\left(d^{4}\right)$ of the substitution impurity. It becomes magnetically active only in $n$ doped materials, e.g., in $(\mathrm{Ga}, \mathrm{Mn}) \mathrm{N}$, where part of the Mn impurities capture donor electrons and transform into charged ions $\mathrm{Mn}^{2+}\left(d^{5}\right)$. A similar behavior is characteristic of all other light TM ions in all III-V compounds including $\mathrm{GaAs}$ and $\mathrm{GaP}^{27,28,35}$

Since the position of the valence band in $\mathrm{GaAs}$ and $\mathrm{GaP}$ is substantially higher (with the vacuum energy as the reference point) than those in GaN, the CFR $t_{2}$ level $\left(d^{5} / d^{4}\right)$ in the (Ga,Mn)As and (Ga,Mn)P DMS's falls below the center of gravity of the valence band. Therefore, this CFR $t_{2}$ level is always occupied, $\mathrm{Mn}$ ions retain five electrons in the $3 d$-shell, and the neutral substitution impurity state is $\left(3 d^{5} \bar{p}\right)$, where $\bar{p}$ stands for a loosely bound hole on the relatively shallow acceptor level in the forbidden energy gap of GaAs and $\mathrm{GaP}$ semiconductors.

Both these situations may be described within the resonance scattering model of the TM impurity states in semiconductors based on the Anderson single impurity Hamiltonian for TM impurities in metals, ${ }^{32}$ and later modified for semiconductors in Refs. 25, 26, 36, 38, and 39. An extension of the Anderson model to two TM impurities in metals was proposed in Ref. 33, which resulted in a FM coupling in TM doped metals and in an interaction similar to the RKKY exchange. ${ }^{45}$ Here we discuss interaction between Mn (and other TM) impurities in a semiconductor host. According to the Hund rule for $\mathrm{Mn}$ ions in a tetrahedral environment, ${ }^{27,28,46}$ two competing states $\mathrm{Mn}^{3+}\left(d^{4}\right)$ and $\operatorname{Mn}^{2+}\left(d^{5}\right)$, involved in the double exchange in DMS, have the $d^{4}\left(e^{2} t^{2}\right)$ and $d^{5}\left(e^{2} t^{3}\right)$ configurations, respectively. The next charged state $d^{6}\left(e^{3} t^{3}\right)$ has a much higher energy due to the strong intra-ionic Coulomb interaction. Therefore, the "passive" nonbonding $e$-electrons may be excluded from our consideration. The indirect exchange between magnetic moments is mediated by virtual transitions of $t_{2}$ electrons via unoccupied valence band states.

\section{B. Model Hamiltonian and impurity related energy}

The model Hamiltonian for two TM impurities in a III-V semiconductor has the form:

$$
H=H_{h}+H_{d}+H_{h d},
$$

where the band Hamiltonian

$$
H_{h}=\sum_{\mathbf{p}, \sigma} \varepsilon_{\mathbf{p}}^{h} c_{\mathbf{p} h \sigma}^{\dagger} c_{\mathbf{p} h \sigma}
$$

includes only the heavy hole band. Here $c_{\mathbf{p} h \sigma}^{\dagger}\left(c_{\mathbf{p} h \sigma}\right)$ is the creation (annihilation) operator of a hole with momentum $\mathbf{p}$ and spin orientation $\sigma$ in the $h h$ band of the semiconductor with the energy dispersion $\varepsilon_{\mathbf{p}}^{h}$. The heavy hole band gives the dominant contribution to the formation of the impurity states, ${ }^{27,28}$ and governs the onset of FM order. The second term in the Hamiltonian (1) describes electrons within the $\mathrm{Mn}$ atoms with an account of the crystal field of the surrounding atoms. In principle, one needs a multielectron Hamiltonian for the degenerate states of the $d$ shell, including Coulomb and exchange interactions. However, it is sufficient to consider the nondegenerate Hamiltonian

$$
H_{d}=\sum_{i \sigma}\left(E_{d} \hat{n}_{i \sigma}+\frac{U}{2} \hat{n}_{i \sigma} \hat{n}_{i-\sigma}\right),
$$

which simplifies the calculation considerably. Here $E_{d}$ is the atomic energy level of the localized $\mathrm{Mn} t_{2}$-electrons and $U$ is the Anderson-Hubbard repulsive parameter; $\hat{n}_{i \sigma}=d_{i \sigma}^{\dagger} d_{i \sigma}$ is the occupation operator for the manganese $t_{2}$-electrons on the impurities, labeled $i=1,2$ in (III, Mn)V DMS. The situations when the degeneracy of the $t_{2}$ states is important and the exchange interaction (Hund rule) in the multielectron atom plays an essential part will be outlined and the appropriate corrections will be introduced, when necessary.

The last term in Eq. (1) describes scattering of heavy holes by the impurities

$$
\begin{gathered}
H_{h d}=H_{h d}^{(r)}+H_{h d}^{(p)}, \\
H_{h d}^{(r)}=\sum_{\mathbf{p}, \sigma, j}\left(V_{\mathbf{p} d} c_{\mathbf{p} h \sigma}^{\dagger} d_{j \sigma} e^{i \mathbf{p} \mathbf{R}_{\mathbf{j}} / \hbar}+\text { h.c. }\right), \\
H_{h d}^{(p)}=\sum_{\mathbf{p} \mathbf{p}^{\prime}, \sigma, j} W_{\mathbf{p p}^{\prime}} c_{\mathbf{p} h \sigma^{\dagger} c_{\mathbf{p}^{\prime} h \sigma} e^{i\left(\mathbf{p}-\mathbf{p}^{\prime}\right) \mathbf{R}_{\mathbf{j}} / \hbar} .}
\end{gathered}
$$

Here $V_{\mathbf{p} d}$ is the $p$ - $d$ hybridization matrix element, and $W_{\mathbf{p} \mathbf{p}^{\prime}}$ is the scattering matrix element due to the difference of the 
pseudopotentials of the host and the substituted atoms. Direct overlaps between the $d$-electron wave functions of the neighboring impurities in the DMS and the Coulomb interaction between them are neglected.

The neglected above orbital degeneracy of the $t_{2}$ states is important at least in three respects. First, the half-filled $d^{5}$ subshell of Mn is occupied by $e$ and $t_{2}$ electrons with parallel spins in accordance with the Hund rule, so the sixth electron can be captured in the $d^{6}$ configuration only with the opposite spin. The energy cost of this capture is $\sim U+J$, where $J \ll U$ is the exchange energy. This feature of the impurity is preserved in the above simplified Hamiltonian (3): the reaction $d^{5}+e \rightarrow d^{6}$ is changed for $d^{1}+e \rightarrow d^{2}$, with the spin of the second electron opposite to the first one. The energy cost of this reaction is $U$, and the principal features of the ion, important for the formation of the localized magnetic moment, are practically the same as in the original atom.

Second, it is the Hund rule that requires that the total angular moments of the $\mathrm{Mn}$ atoms be parallel to allow an indirect inter-impurity interaction between the high-spin $d^{5}$ states via the $h h$ valence band states. We will take this requirement into account, when calculating $T_{C}$, and obtain the energy gain due to the indirect interaction, monitored by the Hund rule, which leads eventually to FM ordering in DMS's.

Third, the threefold degeneracy of the $t_{2}$ electrons is also manifested in the statistics of the localized states, and therefore it influences the position of the Fermi energy in the energy gap. The degeneracy introduces also numerical factors in the effective exchange constants calculated in the Appendix.

Now we proceed with the derivation of indirect interimpurity exchange. To calculate the energy of the exchange interaction between two magnetic impurities, one should find the electronic spectrum of the semiconductor in the presence of two impurities. Each impurity perturbs the host electron spectrum within a radius $r_{b}$. Interimpurity interaction arises, provided the distance between the impurities $R_{i j}$ is comparable with $2 r_{b}$. General equations for the two-impurity states and the corresponding contribution to the exchange energy are derived in the Appendix. Here we present only the final equations, to be used in the derivation of the effective exchange coupling and the Curie temperatures for the specific DMS's. We actually need the impurity related correction to the energy of the system, which is given by the standard formula (see, e.g., Refs. 26 and 45)

$$
\begin{aligned}
E^{\mathrm{magn}}= & \frac{1}{\pi} \operatorname{Im} \int_{-\infty}^{\infty} \varepsilon \cdot \operatorname{Tr} \Delta \mathrm{G}[\varepsilon-i \delta \operatorname{sign}(\varepsilon-\mu)] d \varepsilon \\
& -\sum_{i} U n_{d i \uparrow} n_{d i \downarrow} .
\end{aligned}
$$

$\Delta G=G(\varepsilon)-g^{0}(\varepsilon)$ is the two impurity correction to the total one-particle Green function $\mathrm{G}(\varepsilon)=(\varepsilon-\mathrm{H})^{-1}$.

Here $\mathbf{G}$ is defined as a matrix in $(h, d)$ space, and $\mathrm{g}^{0}$ is the same matrix in the absence of impurity scattering, see Eq. (A7). The Green function is calculated in Appendix in the self-consistent Hartree-Fock approximation for the interimpurity electron-electron repulsion $U$, which is sufficient for a description of magnetic correlations in TM impurities in semiconductors. Then the part of the two-impurity energy (5) responsible for the interimpurity magnetic interaction may be found [see Eqs. (A16) and (A17)]. According to Refs. 25 and 38 , the resonance scattering alone may result in CFR and DBH levels split off from the valence band, provided the scattering potential is strong enough. However, in the case of shallow DBH states the potential scattering may dominate their formation. Like in the well-known Koster-Slater impurity model, the potential scattering in our model is described by a short-range momentum independent coupling constant, $W_{\mathbf{p p}^{\prime}} \approx W$, and the same approximation is adopted for the resonance scattering parameter, $V_{\mathbf{p} d} \approx V$.

Then Eq. (5) acquires the compact form

$$
\Delta E=-\frac{1}{\pi} \operatorname{Im} \int_{\varepsilon_{h b}}^{\varepsilon_{F}} d \varepsilon\left[\ln \mathrm{R}^{\sigma}(\varepsilon)+\ln \mathrm{Q}^{\sigma}(\varepsilon)\right]+\Delta E_{\mathrm{loc}}(\varepsilon<\mu) .
$$

Here the first term in the r.h.s. describes the band contribution to the exchange energy, and the integration is carried out from the bottom of the $h h$ band $\left(\varepsilon_{h b}\right)$ to the Fermi level $\varepsilon_{F}$. The kinetic energy gain of the band electrons due to scattering by FM aligned impurities is incorporated in this integral. The contributions of resonance and potential scatterings are given by the first and second terms, respectively. The last term $\Delta E_{\mathrm{loc}}$ is the contribution of the occupied localized CFR and/or DBH states. These states are described by zeros of the functions $R(\varepsilon)$ and $Q(\varepsilon)$ in the discrete part of the energy spectrum [see Eqs. (A10) and (A8), respectively].

\section{Exchange via heavy hole band states}

The energy lowering results from the indirect spindependent interimpurity overlap, and the mechanism of the effective exchange interaction may be qualified as a kinematic exchange. The double exchange favors a FM order, because splitting of the energy levels belonging to two adjacent impurities occurs due to electron exchange via unoccupied band or impurity-related states without spin reversal. Were the impurity angular moments nonparallel, the Hund rule would suppress the probability of the electron, with the spin parallel to the first impurity angular momentum, to participate in the exchange with the second impurity with a "wrong" direction of the angular moment.

The level splitting, when not suppressed by the Hund rule, results in an energy decrease provided that not all of the available band and impurity levels are occupied. One should note that this kinematic exchange cannot be reduced to any conventional double exchange mechanism because of an actual interplay between three contributions to the magnetic energy, namely, the scattered valence band electrons, the CFR states and the DBH states. We start with the analysis of the band contribution $\Delta E_{b, \mathrm{ex}}$. Since in all the cases we deal with nearly filled $h h$ bands, it is convenient to calculate the energy in the hole representation. By means of a simple trick, described in the Appendix, the contribution of the $h h$ band in the basic equation (6) may be transformed into 


$$
\Delta E_{b, e x}=\frac{1}{\pi} \operatorname{Im} \int_{\varepsilon_{F}}^{\varepsilon_{h t}} d \varepsilon \ln \frac{\mathrm{R}(\varepsilon)}{\mathrm{R}_{0}(\varepsilon)},
$$

where $\varepsilon_{h t}$ stands for the top of the valence band. This equation follows from (A19) by neglecting the potential scattering in the band continuum. Then, inserting Eq. (A10) for the matrix $\mathrm{R}(\varepsilon)$ we obtain

$$
\begin{aligned}
\Delta E_{b, e x} & \\
= & -\frac{1}{2 \pi} \operatorname{Im} \int_{\varepsilon_{F}}^{\varepsilon_{h t}} d \varepsilon \\
& \times \ln \left\{\frac{\left[g_{d}^{-1}(\varepsilon-i \delta)-V^{2} L_{11}(\varepsilon-i \delta)\right]^{2}-V^{4} L_{12}^{2}(\varepsilon-i \delta)}{g_{d}^{-1}(\varepsilon-i \delta)-V^{2} L_{11}(\varepsilon-i \delta)}\right\} .
\end{aligned}
$$

Here

$$
L_{i j}(\varepsilon-i \delta) \equiv P_{i j}(\varepsilon)+\frac{i}{2} \Gamma_{i j}(\varepsilon)
$$

are the lattice Green functions (A9) of the continuous energy argument $\varepsilon$. Retaining only the leading (quadratic) terms in $L_{12}$ in Eq. (8), the exchange energy due to the $h h$ band becomes

$$
\Delta E_{b, \mathrm{ex}}=-\frac{V^{4}}{\pi} \int_{\varepsilon_{F}}^{\varepsilon_{h t}} d \varepsilon \frac{P_{12}(\varepsilon) \Gamma_{12}(\varepsilon)}{\left[\varepsilon-E_{d}-V^{2} P_{11}(\varepsilon)\right]^{2}+\frac{V^{4}}{4} \Gamma_{11}^{2}(\varepsilon)} .
$$

Equation (8) with $L_{12}(\varepsilon) \neq 0$ holds only if the impurity angular moments are parallel to each other (FM case). Then the Hund rule allows for an electron exchange, which takes place without spin-flips. Otherwise the Hund rule blocks the exchange with the neighboring impurity with a "wrong" angular momentum direction. This is accounted for by assuming $L_{12}=L_{21}=0$. Thus, Eq. (10) represents the difference between the states of two impurities with parallel and nonparallel angular moments.

\section{Exchange due to localized impurity states}

Another contribution to the magnetic energy $\Delta E$ [Eq. (6)] originates from the interimpurity exchange due to empty localized states, if available. As was mentioned above, there may be two types of such states, namely CFR and DBH-type levels. For the CFR states, one may, to a good approximation, neglect the potential impurity scattering. To take properly into account the Coulomb blockade effect on the impurity site, one has to calculate the Green function in a selfconsistent way, known as "Hubbard I" approximation [see Eqs. (A14) and (A15) in the Appendix].

The origin of the energy lowering is the inter-impurity level splitting derived in the Appendix [Eqs. (A20) and (A21)]

$$
\delta E_{\mathrm{CFR} \pm}= \pm \Delta+\frac{9 K^{2}}{2} \frac{V^{4}}{\left[1-K V^{2} P_{11}^{\prime}\right]^{2}} \frac{d P_{12}^{2}}{d \varepsilon},
$$

where the splitting of the levels of the isolated impurities is

$$
\Delta(\mathbf{R})=\frac{K V^{2} P_{12}(\mathbf{R})}{1-K V^{2} P_{11}^{\prime}} .
$$

The second term in Eq. (11) results from the repulsion of the two-impurity levels from the band continuum. All the functions $P_{i j}$ and their derivatives $P_{i j}^{\prime}$ are taken at $\varepsilon=E_{\mathrm{CFR}}^{0}$, i.e. at their positions for the isolated impurities (A20).

We may now apply the procedure outlined in Appendix and find the kinematic exchange due to the DBH states above the top of the valence band

$$
\Delta E_{\mathrm{DBH}, \mathrm{ex}}=\frac{K V^{2} P_{12}^{2}}{\left(E_{\mathrm{DBH}}^{0}-E_{d}-V^{2} P_{11}\right) P_{11}^{\prime}} .
$$

All the functions in Eq. (13) should be calculated at $\varepsilon$ $=E_{\mathrm{DBH}}^{0}$. This part of the kinematic exchange favors the FM ordering, since $P_{11}^{\prime}\left(E_{\mathrm{DBH}}^{0}\right)<0$. If the DBH levels lie not too far from the top of the valence band their contribution is comparable with that of the hole pockets and should be properly taken into account when calculating the Curie temperature. At a sufficiently high Mn content the splitting of the DBH level results in formation of an impurity band and its merging with the $h h$ band.

The contribution, $\Delta E_{\mathrm{loc}}$, of the localized states to the kinematic exchange is not universal. It depends on the type of conductivity and should be considered separately for $p$ - and $n$-type materials. Empty shallow Mn-related levels are present in $p-(\mathrm{Ga}, \mathrm{Mn}) \mathrm{As}$ and $p-(\mathrm{Ga}, \mathrm{Mn}) \mathrm{P}$ and apparently absent in $p$-(Ga,Mn)N. In $n$-type $(\mathrm{Ga}, \mathrm{Mn}) \mathrm{N}$, the localized states are due to the deep Mn CFR levels, and the exchange via these levels alone determines the whole mechanism. The double exchange mechanisms in these two cases are obviously different.

\section{MAGNETIC ORDER IN $p$-TYPE DMS}

Here we discuss formation of FM order in Mn-doped III-V p-type semiconductors, of which $(\mathrm{Ga}, \mathrm{Mn}) \mathrm{As}$ and $(\mathrm{Ga}, \mathrm{Mn}) \mathrm{P}$ are the most celebrated. CFR d-like levels lie in these systems below the heavy hole band. DBH levels formed above the top of the valence band broaden into impurity band, and merge with the valence band. The band structure of $(\mathrm{Ga}, \mathrm{Mn})$ As is shown schematically in Fig. 1.

\section{A. Heavy hole and impurity band merging}

The empty localized levels above the Fermi energy appear due to the combined action of both the potential $(W)$ and resonance $(V)$ scattering mechanisms. While fitting the theory to experimental data we use the Fermi energy $\varepsilon_{F}$ instead of the chemical potential $\mu$, since they nearly coincide under the conditions of the experiment. These levels are found as zeros of the determinant $\mathrm{R}(\varepsilon)$ [see Eq. (A10)]. Neglecting small corrections due to resonance scattering, the energy of an isolated DBH level, $E_{\mathrm{DBH}}^{0} \equiv \varepsilon_{i}$, corresponds to a zero of the function

$$
q\left(\varepsilon_{i}\right)=1-W P_{11}\left(\varepsilon_{i}\right)=0,
$$

like in the Koster-Slater one-impurity problem (see the graphic solution on the left panel of Fig. 1). We assume 


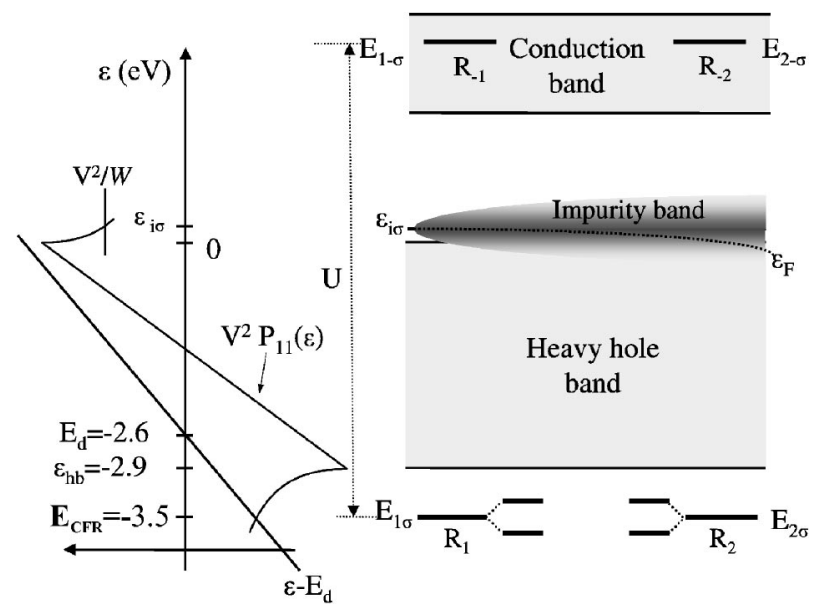

FIG. 1. Left panel: graphical solution of Eq. (18) for the bonding CFR and Eq. (14) for the antibonding DBH levels. Right panel: energy levels in (Ga,Mn)As. The CFR $d$-levels $d^{5} / d^{4}$ (denoted by $\left.R_{1(2)}\right)$ of each impurity, lie below the $h h$ band. The DBH levels (energies $\varepsilon_{1 \sigma}, \varepsilon_{2 \sigma}$ ) are split from the $h h$ band and form localized (acceptor) levels in the energy gap. The CFR levels $d^{6} / d^{5} R_{-1(-2)}$ lie high in the conduction band. The impurity band is shown by the shaded area together with the position of the Fermi level as a function of the width of the impurity band (horizontal axis).

below the model semielliptic density of states for the $h h$ band

$$
\rho(\varepsilon)=\frac{2}{\pi w^{2}} \sqrt{w^{2}-4\left(\varepsilon+\frac{w}{2}\right)^{2}},
$$

where $w$ is the $h h$ bandwidth, and the energy $\varepsilon$ is counted from the top of the $h h$ band. Then

$$
\varepsilon_{i}=E_{\mathrm{DBH}}^{0}=W\left(1-\frac{w}{4 W}\right)^{2} .
$$

For $w=2.9 \mathrm{eV}$ (Ref. 47) and $W=1.02 \mathrm{eV}$, one obtains $\varepsilon_{i}$ $=85 \mathrm{meV}$ for $(\mathrm{Ga}, \mathrm{Mn}) \mathrm{As}$. The influence of resonance scattering on the positions of the shallow DBH levels of the two interacting impurities may be usually neglected (see Ref. 38 for a detailed description of the interplay between resonance and potential impurity scattering). At a sufficiently high impurity content, $x$, an impurity band is formed in $(\mathrm{Ga}, \mathrm{Mn}) \mathrm{As}$ since the DBH levels are split due to the indirect interaction within the impurity pairs,

$$
\delta \varepsilon_{ \pm}=-\frac{1}{W P_{11}{ }^{\prime}} \mathrm{q}_{ \pm},
$$

where $q_{ \pm}$are the two solutions of Eq. (A23).

Then Eqs. (16) and (A23) result in

$$
\Delta_{\mathrm{DBH}}(\mathbf{R}) \equiv \frac{1}{2}\left(\delta \varepsilon_{+}-\delta \varepsilon_{-}\right)=-\frac{P_{12}(\mathbf{R})}{P_{11}{ }^{\prime}} .
$$

The functions $P_{i j}$ and $P_{i j}^{\prime}$ are calculated at $\varepsilon=\varepsilon_{i}$. The halfwidth of the impurity band can be roughly estimated as $z \Delta_{\mathrm{DBH}}(\bar{R})$, where $\bar{R}$ is the typical distance between the impurities. The $z$ value characterizes the number of neighboring impurities participating in the interaction with any given impurity. At $z \Delta_{\mathrm{DBH}}(x)>\varepsilon_{i}$ the impurity band merges with the valence band and they both form a unified continuum of states. For the above values of the model parameters this merging occurs even at $x<0.01$.

\section{B. Double exchange in $p$-type DMS}

Eventually, magnetic order arises due to the exchange interaction between the occupied CFR levels. These levels correspond to the states $d^{5} / d^{4}$ of the Mn ions below the $h h$ band, whereas the empty $d^{6} / d^{5}$ CFR levels are pushed up to the conduction band by the Anderson-Hubbard repulsion $U$ (Fig. 1). The left panel of this figure depicts a graphical solution of the equation

$$
\mathrm{R}(\varepsilon)=0,
$$

with $\mathrm{R}(\varepsilon)$ defined in Eq. (A10). Neglecting the interaction between the impurities, $L_{12}=0$ [and, hence $M_{12}(R)=0$ ], Eq. (17) takes the form

$$
E_{\mathrm{CFR}} \equiv E_{i \sigma}=E_{d}+V^{2} L_{i i}\left(E_{i \sigma}\right)
$$

and describes the formation of the deep impurity states of CFR type out of the atomic $d$-levels $E_{d}$ below the $h h$ valence band. The values of the parameters used in the graphical solution presented in Fig. 1 will be discussed below.

FM order arises, provided the state with the parallel impurity angular moments is energetically preferable in comparison to that with the nonparallel moments. The splitting of the CFR levels due to nonzero $L_{12}(R)$ shown in the right panel of Fig. 1 cannot give an energy gain, when both states are occupied. One should take into account all the changes in the energy spectrum, namely, the reconstruction of the partially filled merged impurity and $h h$ bands. The indirect interaction involving empty states near the top of the $h h$ band is in fact a novel type of the double FM exchange, which resembles the well known Zener exchange ${ }^{30}$ but differs from it in many important aspects (see below).

The energy diagram presented in Fig. 1 is confirmed by numerical LDA-CPA calculations. ${ }^{24}$ One finds in the spinpolarized density of states both occupied CFR-like $t_{2}$ majority spin component and empty $t_{2}$ minority spin component. The up-spin hole pocket of the same $t_{2}$ symmetry is formed by $\mathrm{DBH}$-like states near the top of the valence band merged with the shallow impurity band.

The contribution favoring the FM order can be obtained from Eq. (10) with the addition of the part due to the impurity band merged with the valence band,

$$
\Delta E_{\mathrm{FM}}=-\frac{V^{4}}{\pi} \int_{\varepsilon_{F}(x)}^{\varepsilon_{i}+z \Delta_{\mathrm{DBH}}(x)} d \varepsilon \frac{\Gamma_{12}(\varepsilon) P_{12}(\varepsilon)}{\left[\varepsilon-E_{d}-V^{2} P_{11}(\varepsilon)\right]^{2}+\frac{V^{4}}{4} \Gamma_{11}^{2}(\varepsilon)} .
$$

In the FM-aligned spin configuration only the majority spin subband contributes to $\Delta E_{\mathrm{FM}}$, therefore the spin index is omitted for the sake of brevity.

The approximations used in calculating the lattice Green function $L_{i j}(R)$ with the interimpurity distance $R$ are de- 
scribed in Eq. (8) of Ref. 29. However, now the impurity band neglected in Ref. 29 plays an important part. It merges with the top of the valence band of $(\mathrm{Ga}, \mathrm{Mn}) \mathrm{As}$ at the concentrations above $x_{\text {crit }}=0.0065$ and contributes to creating FM order. Earlier transport measurements for low $T_{C}$ samples indicate merging even at $x=0.002$. All this justifies the approximation adopted for the hole states in Eq. (19). This equation is our working formula, from which we obtain $T_{C}$. It has been also demonstrated in Ref. 29 that the antiferromagnetic (AFM) Anderson-type superexchange ${ }^{33}$ cannot compete with the FM double exchange.

Now the difference between the double exchange mechanism in DMS and the Zener double exchange in transition metal oxides becomes clear. The latter was proposed ${ }^{30}$ for $\left(\mathrm{La}, \mathrm{A}^{2+}\right) \mathrm{MnO}_{3}$ where $\mathrm{Mn}$ ions were in different valence states $\left(\mathrm{Mn}^{3+}\right.$ and $\left.\mathrm{Mn}^{4+}\right)$. In our case it would have meant that one of the two levels $E_{\mathrm{CFR} \uparrow}$ were empty. As seen from Fig. 1 these states in (Ga,Mn)As are both occupied, and the Zener mechanism in its original form is not applicable. Actually, the FM order in the $p$-type DMS arises due to the kinematic exchange between the two $\operatorname{Mn}\left(d^{5}\right)$ ions via the empty states near the top of the valence band.

\section{Fitting procedure: (GaMn)As}

The dependence of the Fermi level on the Mn content $\varepsilon_{F}(x)$ is found from the equation

$$
x_{s}=2 \int_{\varepsilon_{F}(x)}^{\delta w(x)} \rho(\varepsilon) d \varepsilon,
$$

in which $\delta w(x)=\varepsilon_{i}+z \Delta_{\mathrm{DBH}}(x)$, and the $h h$ density of states is approximated as

$$
\begin{aligned}
\rho(\varepsilon)= & 8 /\left[\pi(w+\delta w(x))^{2}\right] \sqrt{[\delta w(x)-\varepsilon](\varepsilon+w)} \\
& \times \theta[\delta w(x)-\varepsilon] \theta(\varepsilon+w) .
\end{aligned}
$$

The per site hole concentration $x_{s}$ is proportional to the hole density $p_{h}\left(x_{s}=a^{3} p_{h} / 8\right.$ in the zinc-blende structure). Equations (19) and (20) allow one to determine the pair exchange energy as a function of $\mathrm{Mn}$ concentration $x$ and connect it with the experimental data for the given hole concentrations $p_{h}(x)$ which are taken from the measurements. ${ }^{48}$

The Curie temperature $T_{C}$ was calculated in the molecular field approximation. According to this approach the spin Hamiltonian reads

$$
H_{\mathrm{MF}}=\frac{1}{2} \sum_{i} \Delta E_{\mathrm{FM}}\left(\mathbf{R}_{i j}\right) \mathbf{J}_{i} \cdot\langle\mathbf{J}\rangle,
$$

where summation runs over all positions of the Mn impurities with an angular moment operator $\mathbf{J}_{i}$. Here the factor $1 / 2$ accounts for the fact that the energy gain of the FM vs AFM orientation of two coupled spins is $\Delta E_{\mathrm{FM}}\left(\mathbf{R}_{i j}\right)$, contrary to $2 J_{\text {Heis }}$ in the Heisenberg model. Then $T_{C}$ is readily found,

$$
T_{C}=-\frac{\Delta E_{\mathrm{FM}}(x)}{k_{B}} \frac{\bar{z} J(J+1)}{6},
$$

where $\bar{z}$ is, similarly to $z$, a measure of the number of neighboring atoms participating in the exchange interaction. ${ }^{49}$ It is

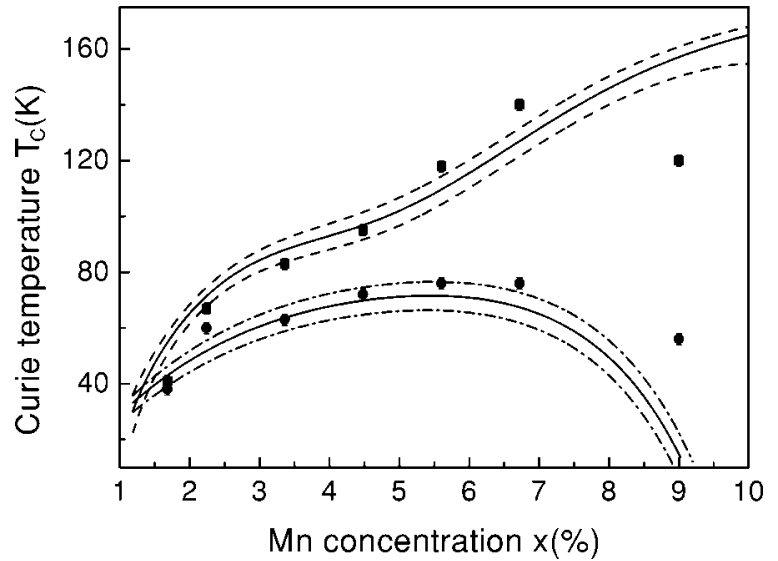

FIG. 2. Calculated dependence of $T_{C}$ on the manganese concentration $x$ in $(\mathrm{Ga}, \mathrm{Mn})$ As based on the experimental data for the hole density (Ref. 48) $p_{h}(x)$. Solid squares (circles) stand for experimental $T_{C}(x)$ of annealed (as-grown) samples. Broken lines take into account the error bars of the hole density.

certainly close to $z$, although does not necessarily coincide with it. In $(\mathrm{Ga}, \mathrm{Mn})$ As the total angular moment of a complex $\operatorname{Mn}\left(3 d^{5} \bar{p}\right)$ is unity: ${ }^{41} J=1$, since it is formed by the moment $j=3 / 2$ of the loosely bound hole AFM-coupled to the Mn center with the spin $S=5 / 2$. Then the numerical factor in Eq. (22) is close to unity. The results of our calculations are presented in Figs. 2 and 3.

In order to find the dependence $T_{C}(x)$, the value of the Fermi level, Eq. (20) calculated from the experimental date, ${ }^{48}$ is used in Eqs. (22) and (19). A polynomial fit is used for the hole density $p_{h}(x)$ in Eq. (19). The values of the model parameters characterizing the impurity $d$-state are $U$ $\approx 4.5 \mathrm{eV}, V=1.22 \mathrm{eV}$, while the $h h$ mass $m=0.51 \cdot m_{0}$ and $h h$ bandwidth $w=2.9 \mathrm{eV}$ were taken from Ref. 47. The hybridization strength $V$ was obtained from Eq. (18) with $E_{\mathrm{CFR}}=$ $-3.47 \mathrm{eV}\left(E_{\mathrm{CFR}}^{\exp }=-3.4 \mathrm{eV}\right.$ according to Ref. 50). The energy of the shallow acceptor level was chosen to be $\varepsilon_{i}=85 \mathrm{meV}$,

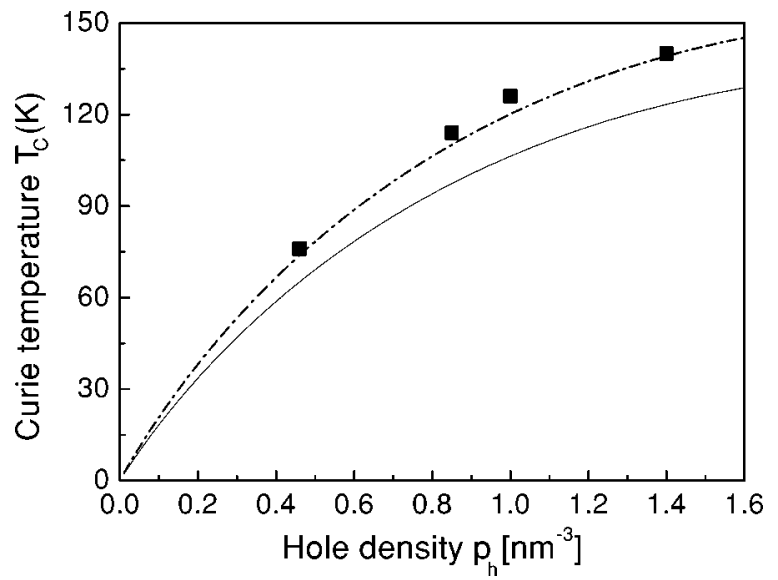

FIG. 3. The dependence of the Curie temperature on the hole density $p$ in $(\mathrm{Ga}, \mathrm{Mn})$ As. Solid and dashed-dotted lines are the theoretical curves for the two values of the hybridization parameter $(V=1.22$ and $1.24 \mathrm{eV}$, respectively). Experimental points (filled squares) are taken from Ref. 48. 


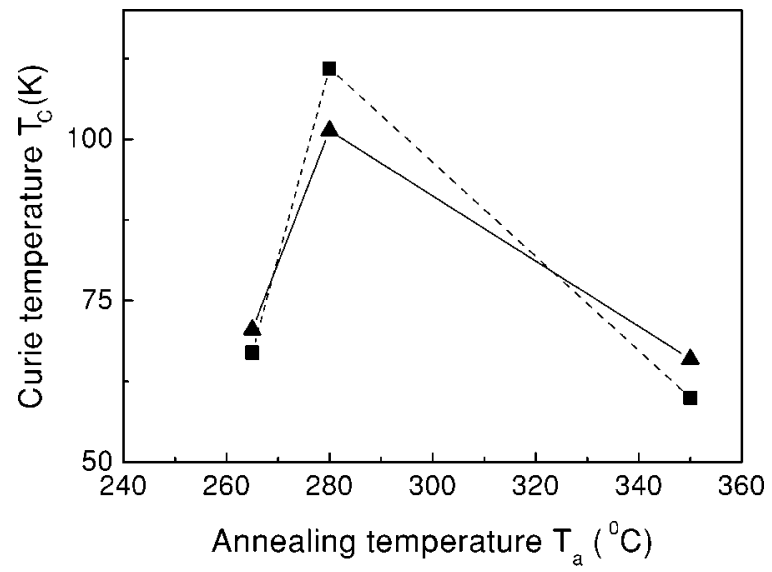

FIG. 4. Calculated $T_{C}$ (triangles connected by a solid line) vs the annealing temperature $T_{a}$ in $(\mathrm{Ga}, \mathrm{Mn}) \mathrm{As}$. The data for the hole density and the experimental values of $T_{C}\left(T_{a}\right)$ (closed squares) are taken from Ref. 51. The parameters used in the calculations are: $V=1.22 \mathrm{eV}, \bar{z}=4.0, E_{\mathrm{CFR}}=-2.6 \mathrm{eV}$.

close the experimental value ${ }^{28} \varepsilon_{i}^{\text {exp }}=110 \mathrm{meV}$. The value of $\bar{z}=2.5$ was taken for the coordination number in as-grown samples (Fig. 2, lower curve). At these values for the model parameters the ratio $\left|\Delta E_{\mathrm{FM}}\right| /\left|\Delta E_{\mathrm{AFM}}\right| \sim 2$ justifies the dominance of the FM coupling in $(\mathrm{Ga}, \mathrm{Mn}) \mathrm{As}$.

The nonmonotonous dependence of $T_{C}(x)$ is due to a nonequilibrium character of the sample preparation. Apparently, the ratio between the concentration of Mn impurities and the actual hole concentration depends on the doping method and the thermal treatment. In particular, the annealing of the sample results in a reduction of the donorlike Mn-related interstitial defects in favor of acceptorlike substitution impurities. ${ }^{48,51}$ To describe the annealing effect we changed the value of $\bar{z}$ from 2.5 to 4 in Eqs. (19) and (22). The results of numerical fitting are shown in the upper curve of Fig. 2.

Recent detailed measurements of the hole concentrations in a series of both as-grown and annealed $\mathrm{Ga}_{1-x} \mathrm{Mn}_{x} \mathrm{As}$ samples, ${ }^{48}$ allowed us to compare the theoretical plot $T_{C}(p)$ with the experimental data (see Fig. 3). The same equations (22) and (19), and the same values for the model parameters $E_{\mathrm{CFR}}, U, \varepsilon_{i}$ as in the above estimate are used. The Mn concentration $x=0.067$ is taken as the reference point, and the coordination number being $\bar{z}=4$. Two theoretical curves correspond to two values of the hybridization parameter $V$ $=1.22$ and $1.24 \mathrm{eV}$ (solid and dashed-dotted curves, respectively). One may conclude from these two fittings that the theory is rather sensitive to the choice of the model parameters. To check the stability of our description we made one more fitting of the experimental data obtained only for annealed samples (see Fig. 4). These data are taken from Ref. 51. The same set of model parameters gives satisfactory quantitative agreement with the experiment for these samples as well.

\section{D. (GaMn)P}

Thus, our theory provides a satisfactory quantitative description of the behavior of the Curie temperature $T_{C}$ as a function of the Mn content $x$ and the hole concentration $p_{h}$ in $p$-(Ga, Mn)As. This description is applicable also to $p$ - $(\mathrm{Ga}, \mathrm{Mn}) \mathrm{P}$. The band structure of $(\mathrm{Ga}, \mathrm{Mn}) \mathrm{P}$ can be also schematically represented by Fig. 1, however, the DBH level lies deeper in the energy gap than in $p-(\mathrm{Ga}, \mathrm{Mn}) \mathrm{As}$ : its position is estimated as $\approx 0.4 \mathrm{eV}$ above the top of the valence band (see, e.g., Refs. 27, 28, 44, and 47). The impurity band appears to be narrower than in $(\mathrm{Ga}, \mathrm{Mn}) \mathrm{As}$. It does not merge with the $h h$ band at any reasonable Mn concentration and its contribution to the indirect exchange can be neglected. We tested our theory by fitting the experimental value ${ }^{52}$ of $T_{C}$ $=270 \mathrm{~K}$ reported for a $p$-type $(\mathrm{Ga}, \mathrm{Mn}) \mathrm{P}$ at $x=0.054$. The impurity level $\varepsilon_{i}=400 \mathrm{meV}$ arises at $W=1.4 \mathrm{eV}$. We have found that the value of $T_{C}=270 \mathrm{~K}$ is reproduced by means of Eq. (22) with the following parameters: $w=2.6 \mathrm{eV}, V$ $=1.31 \mathrm{eV}, \quad x=0.05, \quad \bar{z}=4, \quad E_{d}=-1.3 \mathrm{eV}$ (then $E_{\mathrm{CFR}}=$ $-3.28 \mathrm{eV}$ ). The chosen value of $p_{h}=1 \cdot 10^{20} \mathrm{~cm}^{-3}$ is the hole density in a C-doped $\mathrm{GaP}$ before $\mathrm{Mn}$ implantation. ${ }^{52}$ So the values of our model parameters are close to those for (Ga,Mn)P in the experiments of Ref. 52. Besides, these parameters are in a good correlation with the chemical trends in the systems $(\mathrm{Ga}, \mathrm{Mn}) \mathrm{As},(\mathrm{Ga}, \mathrm{Mn}) \mathrm{P}$ (the latter has a smaller lattice spacing and larger effective mass $m_{h h}^{*}$ than the former).

As for $p$ - $(\mathrm{Ga}, \mathrm{Mn}) \mathrm{N}$, the theory should be modified in order to describe this material. In this case Mn remains a neutral substitution isoelectronic defect in the configuration $\mathrm{Mn}^{3+}\left(d^{4}\right)$, since the $\mathrm{Mn}^{2+/ 3+}$ transition energy falls deep into the wide energy gap in accordance with the experimental ${ }^{44}$ and theoretical ${ }^{23}$ data. If the hole concentration, $p_{h}$, exceeds the Mn content, $x$, then we return to the situation discussed in this section, but with the localized moment $J=2$ characteristic of the $\mathrm{Mn}^{3+}\left(e^{2} t^{2}\right)$ configuration. In the opposite case, the valence band is full, and the Fermi energy lies within the deep impurity band formed by the $\mathrm{Mn}^{2+/ 3+}$ levels. This situation is discussed in the next section.

Unfortunately, there are not enough experimental data on $T_{C}(x)$ available to make a detailed comparison with the theoretical predictions both in $p-(\mathrm{Ga}, \mathrm{Mn}) \mathrm{P}$ and $p-(\mathrm{Ga}, \mathrm{Mn}) \mathrm{N}$ materials. Moreover, no $p$-type conductivity was observed in $(\mathrm{Ga}, \mathrm{Mn}) \mathrm{N}$ even in the cases when the pristine $\mathrm{GaN}$ crystals were of a $p$-type. Due to the lack of experimental information, we have confined ourselves with a quantitative and qualitative description of the $p$-type $(\mathrm{Ga}, \mathrm{Mn}) \mathrm{As}$ and turn now to the case of $n-(\mathrm{Ga}, \mathrm{Mn}) \mathrm{N}$, where the double exchange mechanism, as described above, should be revisited.

\section{MAGNETIC ORDER IN $n$-TYPE DMS}

$\mathrm{Mn}$ is a deep acceptor in GaN, ${ }^{27,28}$ and each Mn impurity creates an empty $t_{2}$ level deep in the energy gap. Therefore, the Fermi energy is pinned to these levels unless other (shallow) acceptors create enough free holes near the top of the valence band. This statement is confirmed by extended cluster "quasiband" calculations. ${ }^{23}$ If a Mn-doped sample contains also shallow donors in a noticeable concentration, then the deep Mn-impurity band is partially filled by electrons, and one arrives at the problem of magnetic order in $n$-type (Ga,Mn)N. In this case the $d^{5} / d^{4}$ CFR energy levels forming 


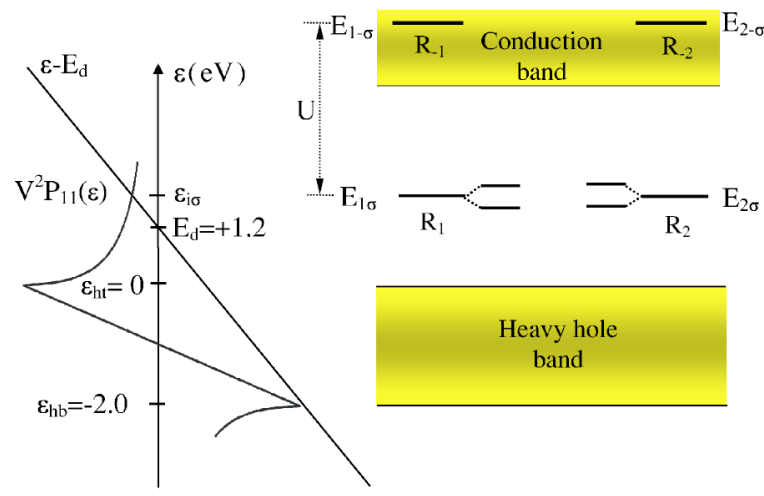

FIG. 5. Left panel: graphical solution of Eq. (18) for the antibonding CFR level in $(\mathrm{Ga}, \mathrm{Mn}) \mathrm{N}$. Right panel: energy levels in $\mathrm{Ga}(\mathrm{Mn}) \mathrm{N}$. The CFR $d$-levels $d^{5} / d^{4}$ (denoted by $R_{1(2)}$ ) of each impurity, lie within the energy gap. The CFR levels $d^{6} / d^{5} R_{-1(-2)}$ lie high in the conduction band.

the impurity band are partially filled, so one encounters the mixed valence $\mathrm{Mn}^{3+} / \mathrm{Mn}^{2+}$ situation similarly to the original Zener model. ${ }^{30}$ Exchange within the impurity band is possible due to an overlap of the tails of the impurity wave functions. These tails are formed by the superpositions of the Bloch electron wave functions from the $h h$ band, so the latter plays in fact the role of the oxygen $p$-orbitals in $\mathrm{La}(\mathrm{Mn}, \mathrm{Sr}) \mathrm{O}_{3}$ considered by Zener.

The Mn impurity band is formed by $d^{5} / d^{4}$ CFR impurity levels, $E_{\mathrm{CFR}}$, which are well separated from the $h h$ band. It may be partially occupied by electrons and be responsible for a magnetization of the DMS. To describe formation of a deep impurity band we solve Eq. (18) for the Mn-related CFR level and then substitute this solution into the secular equation (17) assuming again the semielliptic density of states (15). The bare level $E_{d}$ is now above the top of the $h h$ band, and the $p d$ hybridization pushes it deeper into the energy gap. The graphical solution of Eq. (18) is presented in the left panel of Fig. 5.

The hybridization parameter is nearly the same as in (Ga,Mn)As, $V=1.2 \mathrm{eV}$. The right panel of Fig. 5 illustrates the formation of an impurity dimer via an overlap of the tails of the CFR wave functions (17). Then the overlap of the electron wave functions between the correlated states $R_{1}$ and $R_{2}$ initiates formation of an impurity band and the Zener-type indirect exchange interaction may arise in a partially occupied. Like in $p$-(Ga,Mn)As, our model spectrum correlates with the numerical data obtained by LDA+CPA calculations. ${ }^{24}$ These calculations show an impurity band formed by spin-up CFR states of $t_{2}$ symmetry in the middle of the energy gap and a smaller peak of the same symmetry within the valence band, which may be interpreted as the DBH counterpart of the impurity resonance peak. The empty minority spin $t_{2}$ states arise in the conduction band.

In principle, the double exchange via empty states in the conduction band may also contribute. This contribution is small in zinc blende host crystals, because the hybridization $V_{d s}$ between the impurity $d$-states and the $s$-states of the electrons near the bottom of conduction band is weak due to the symmetry selection rules. ${ }^{27,28}$ However, it may give an additional contribution to the indirect exchange in wurtzite type materials together with the fourth-order in $\left|V_{d p}\right|^{2}\left|V_{d s}\right|^{2}$ Bloembergen-Rowland indirect exchange. ${ }^{53}$ The empty $\left(d^{6} / d^{5}\right)$ CFR levels are pushed up high to the conduction band by the Anderson-Hubbard repulsion $U$. These levels may cause an AFM ordering in the impurity band, but the exchange coupling is relatively weak. We leave more detailed analysis of all these contributions for further studies, because here our main purpose is to establish the impurity band character of magnetism in $n-(\mathrm{Ga}, \mathrm{Mn}) \mathrm{N}$ and to estimate the upper limit of $T_{C}$.

\section{A. Exchange in impurity band}

We consider first a pair of impurities at a distance $R$ from each other. To be definite we assume that the chemical potential $\mu$ lies below the level $E_{\mathrm{CFR}}$ of an isolated impurity. According to Eq. (12) the interaction between the impurities splits the impurity. It is emphasized that the splitting takes place only if the spins $\mathbf{S}_{i}$ of the two impurities are parallel (FM ordering) and the process is not blocked by the Hund rule. The distance $R$ between the impurities should not also exceed a certain value, i.e., $R<R_{\mathrm{ex}}(\mu)$, where $R_{\mathrm{ex}}(\mu)$ is the solution of the equation

$$
\Delta\left(R_{\mathrm{ex}}\right)=E_{\mathrm{CFR}}^{0}-\mu .
$$

Then one of the two impurity levels sinks below the chemical potential $\mu$ and is occupied by an electron, which results in the energy decrease $\Delta(R)$, whereas the second level remains empty and does not contribute to the energy balance. If the spins of the impurities are not parallel, the Hund rule forbids an indirect exchange and there is no energy decrease. Therefore, the exchange energy in such a pair equals just $-\Delta(R)$ for the FM ordering of the spins and to zero otherwise. Then the typical pair exchange interaction can be estimated as

$$
\bar{\Delta}=\int_{0}^{R_{\mathrm{ex}}(\mu)} \Delta(R) g(R) d R,
$$

where $g(R)$ is the impurity pair distribution function.

In order to complete our scheme, we need also an equation connecting the position of the chemical potential with the electron concentration $n$ in the impurity band. This concentration coincides with the concentration of impurities in the $d^{5}$ state, hence

$$
n=\int^{\mu} \rho(\varepsilon) n_{d^{5}}(\varepsilon-\mu) d \varepsilon,
$$

where $\rho(\varepsilon)$ is the density of states in the impurity band. The distribution $n_{d^{5}}(\varepsilon-\mu)$ is calculated in the Appendix, Eq. (A15).

We use below the Fermi energy $\varepsilon_{F}$ of unfilled impurity band instead of $\mu$, as we did for $p$-type samples. At low temperatures $n_{d^{5}}\left(\varepsilon-\varepsilon_{F}\right)$ is 1 for $\varepsilon<\varepsilon_{F}$ and 0 for $\varepsilon>\varepsilon_{F}$. This allows us to circumvent the calculation of the density of states $\rho(\varepsilon)$ and present the condition (25) in the alternative form 


$$
n=\int_{0}^{R_{\mathrm{ex}}\left(\varepsilon_{F}\right)} g(R) d R,
$$

more convenient for our calculations. If an experiment provides us with dependencies of the Curie temperature and the electron concentration on the Mn content, then Eqs. (23) and (26) will allow us to connect the Fermi energy with the electron density in the impurity band. Then Eq. (24) is used to calculate the average pair exchange interaction $\bar{\Delta}(x)$ as a function of the impurity content $x$.

We now appeal to the theory of dilute FM alloys ${ }^{54}$ and arrive at the final result, i.e. the Curie temperature. Unlike the case of $(\mathrm{Ga}, \mathrm{Mn}) \mathrm{As}$, the magnetic energy in impurity band is determined by the spin $S$ of Mn $3 d$ shell, which equals $5 / 2$ and 2 for $d^{5}$ and $d^{4}$ configurations, respectively. In our crude approximation it is sufficient to know that the proportionality coefficient between $T_{C}$ and $\bar{\Delta}$ is close to unity:

$$
T_{C}(x)=\frac{S(S+1)}{6 k_{B}} \bar{\Delta}(x) \approx \bar{\Delta}(x) / k_{B} .
$$

At low electron concentrations, when $R_{\mathrm{ex}}\left(\varepsilon_{F}\right)>d_{0}$, only a minor part of the impurities is coupled by the indirect exchange interaction. The above averaging procedure does not hold any more and a percolation type of approach should be applied. ${ }^{55}$

The behavior of $T_{C}$ for higher electron concentrations, when the Fermi energy lies above the level $E_{\mathrm{CFR}}^{0}$, can be found in a similar fashion. We just have to switch to the hole representation and use the distribution function $3 n_{d^{4}}\left(\varepsilon-\varepsilon_{F}\right)$ instead of $n_{d^{5}}\left(\varepsilon-\varepsilon_{F}\right)$. (Dealing with holes we have now to account for the threefold degeneracy of $t_{2}$ states.) The function $3 n_{d^{4}}$ is 1 if $\varepsilon>\varepsilon_{F}$ and 0 if $\varepsilon<\varepsilon_{F}$. As a result, we will obtain a mirror symmetric dependence of $T_{C}$ on the electron concentration in this range.

\section{B. Curie temperature in $(\mathrm{Ga}, \mathrm{Mn}) \mathrm{N}$}

To get an idea of the behavior of the average kinematic exchange $\bar{\Delta}$ and, hence of the dependence $T_{C}(x, n)$, we make simple estimates. Consider the case when $\varepsilon_{F}<E_{\mathrm{CFR}}^{0}$, then $K=1$ and according to Eq. (A21) the levels of a pair of impurities separated by the distance $R$ are split by

$$
\delta \varepsilon_{ \pm}= \pm \Delta(R)= \pm \frac{V^{2} P_{12}(R)}{1-V^{2} P_{11}^{\prime}}
$$

where the off-diagonal lattice sum can be estimated as

$$
P_{12}(R)=\frac{1}{2 \pi} \frac{m d_{0}^{3}}{\hbar^{2} R} e^{-\kappa R}
$$

Here $\kappa$ is the localization parameter. (Although we use here the estimate $\kappa \approx 1 / \hbar \sqrt{2 m E_{\mathrm{CFR}}^{0}}$, we should not forget that it may be rather crude for really deep levels.) Then

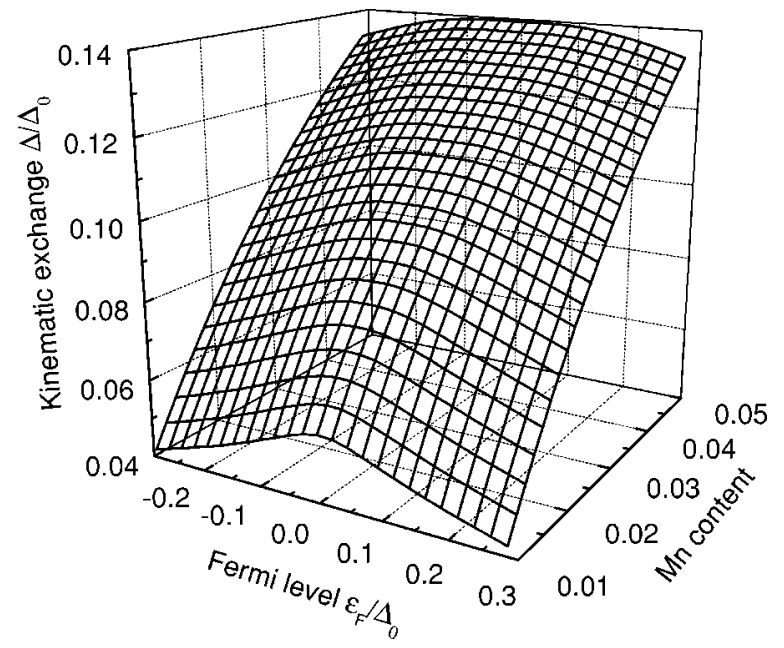

FIG. 6. Dependence of the kinematic exchange, measured in units of $\Delta_{0}$ on the Mn content $(x)$, and on the position of the Fermi energy relative to the level $E_{\mathrm{CFR}}^{0}$ of an isolated $\mathrm{Mn}$ impurity. We took $\kappa^{-1}=d_{0}$.

$$
\Delta(R) \approx \Delta_{0} \frac{d_{0}}{R} e^{-\kappa R},
$$

where the value of $\Delta_{0}$ depends on the parameters of the system but is generally of the order of $1 \mathrm{eV}$.

The pair distribution function in Eq. (24) is modeled by the Poisson pair distribution truncated at small distances $R<d_{0}$,

$$
g(R)=\left\{\begin{array}{cc}
\frac{3 R^{2}}{\bar{R}^{3}} \exp \left\{\frac{d_{0}^{3}-R^{3}}{\bar{R}^{3}}\right\}, & \text { for } R>d_{0}, \\
0, & \text { for } R<d_{0}
\end{array}\right.
$$

where $\bar{R}$ is the average distance between the impurities and $d_{0}$ is the minimal distance between the impurities allowed by the lattice structure, i.e., the distance between two neighboring $\mathrm{Ga}$ atoms.

For a deep enough level we may assume $\kappa \approx 1 / d_{0}$ and calculate the dependence of the average kinematic exchange $\bar{\Delta}$ on the Mn content and position of the Fermi energy with respect to the isolated impurity level $E_{\mathrm{CFR}}$ (see Fig. 6). The two latter quantities are measured in units $\Delta_{0}$. We see that the kinematic exchange and, hence, the Curie temperature is maximal for any given value of $x$ when the impurity band is half filled, i.e., $\varepsilon_{F}=E_{\mathrm{CFR}}^{0}$. At $x=0.05$ the kinematic exchange $\bar{\Delta}$ may be about $0.14 \mathrm{eV}$ (if we assume that $\Delta_{0}=1 \mathrm{eV}$ ). The angular moment $J=2$ for the $d^{4}$ state of the Mn impurity in GaN, hence $T_{C}=\bar{\Delta} / k_{B}$, and we find that the value of the Curie temperature varies from a few hundred $\mathrm{K}$ for $x=0.01$ and may exceed $1000 \mathrm{~K}$ at $x=0.05$. By choosing the localization radius so small, $\kappa^{-1} \approx d_{0}$, we have actually found a lower bound for $\bar{\Delta}$. We can get even higher Curie temperatures for a larger localization radius.

It is emphasized that the averaging procedure used to obtain the results in Fig. 6 works well only when the Fermi energy is close to the midpoint of the impurity band, i.e., 
$E_{\mathrm{CFR}}^{0}$ (zero on the Fermi energy axis). Far from this region the percolation approach ${ }^{55}$ should be used. Then the effective kinematic exchange in this range may become smaller than shown in the figures, however the behavior does not change qualitatively, and certainly remains correct also quantitatively for $\varepsilon_{F}$ close to $E_{\mathrm{CFR}}^{0}$.

To conclude this section, we have found that the optimum $n$-doping level for FM alignment of Mn spins corresponds to the half-filled impurity band, and one may well expect really high temperature magnetism in this case. Unfortunately, lack of information on the $T_{C}$ dependence on the impurity concentration and position of the Fermi level does not allow us to make a more detailed comparison with the experiment.

\section{CONCLUDING REMARKS}

According to the general theory of magnetic interactions, the kinematic exchange between localized impurity spins embedded in a semiconductor host with covalent chemical bonding $^{56}$ is a generic property of the two-impurity cluster. We constructed such a cluster, which was based on an $a b$ initio knowledge about the electronic structure of an isolated Mn impurity in a III-V semiconductor. After some simplifications this approach led us to a microscopic Hamiltonian similar to a two-center Anderson model. ${ }^{33,45}$ That model was originally developed for metallic hosts, whereas we considered here a semiconductor host. The model allows for analytical solutions, and the final equation (22) for $T_{C}$ with the effective coupling constant (19) should be compared with the phenomenological mean-field equation ${ }^{14,57}$

$$
T_{C}=\frac{4 x S(S+1) J_{p d}^{2}}{3 a} \frac{\chi_{h}}{\left(g \mu_{B}\right)^{2}},
$$

where $a$ is the lattice constant, $J_{p d}$ is the phenomenological $p d$-exchange constant, $\chi_{h}$ is the magnetic susceptibility of holes in the valence band. Our Eq. (22) and Eq. (30) consist, in fact, of similar ingredients. It is natural to assume that $J_{p d}$ in the phenomenological model stems from the hybridization. Then it may be derived in the Anderson-type model by means of the usual Schrieffer-Wolff transformation, so that $J_{p d} \sim V^{2} /\left(\varepsilon_{F}-E_{d}\right)$. Looking at Eq. (19) one can readily discern the corresponding contributions as in Eq. (30). (The latter was proposed for dilute magnets four decades ago. ${ }^{58}$ ) The second fraction in Eq. (30) is proportional to $m^{*} k_{F}$, i.e., to the Fermi momentum of holes in the valence band.

Unlike the phenomenological model, which deals with localized spins and free holes near the top of the valence band, our model takes into account the change in the density of hole states (and therefore in their magnetic response) due to resonance scattering and impurity band formation. One of the results of this change is a more complicated dependence of the magnetic coupling on the Mn content $x$ than the linear law predicted by Eq. (30). It is worth noting that the strong $p d$-hybridization is not completely consistent with the Vonsovskii-Zener-type of exchange ${ }^{31}$ used in the phenomenological approach of Refs. 14 and 57. The present model allows one to establish connection between the picture of hybridized $t_{2}$ states and the Heisenberg-type effective spin Hamiltonian.
Another improvement of the mean-field theory, taken into account in Eq. (22), is a more refined description of the impurity magnetic moment. The actual moment $J$ arises as a vector sum of the moments of the $d$-electrons and the bound $p$-hole. One should emphasize that at a high enough concentration all mean-field descriptions fail because the alloy approaches the instability region and the Mn distribution becomes strongly inhomogeneous. In the case of $\mathrm{Ga}_{1-x} \mathrm{Mn}_{x} \mathrm{As}$ this is, apparently, the region of $x>0.07$.

Next, one should discuss the relation between our approach and the so-called $a b$ initio numerical calculations carried out by means of the local-density functional method. Doping by $\mathrm{Mn}$ atoms is modeled in the calculations ${ }^{22,23}$ by means of a finite-size cluster of a GaAs or GaN host with one or several atoms replaced by Mn. A coherent potential technique may be also applied. ${ }^{24}$ Periodic structures are constructed from magnetic clusters ("supercells") and the electronic bands in these artificial objects are calculated in the local-spin-density approximations (LSDA). So, the starting point in our approach and the LSDA calculations is the same. No wonder that the positions of CFR and DBH levels in these calculations are in good agreement with those used in our model, based on previous single impurity calculations. ${ }^{27,28}$ However, a direct comparison of the two procedures, as far as the magnetic properties are concerned, is not straightforward. The self-consistent LSDA method results by construction only in a Stoner-type itinerant magnetism. Therefore, magnetic states are discussed in Refs. 22 and 23 in terms of exchange splitting and majority/minority spin subbands. It is difficult to discern the genuine kinematic double exchange in this type of calculations.

To overcome this difficulty, Sanvito et al. ${ }^{22}$ tried to fit their LDSA calculation to a free-electron model with an effective hole mass $m^{*}$ and uniformly distributed impurities described by a model square potential containing the spinindependent, $W(r)$, and exchange components, $J(r)$. This purely phenomenological separation ignores the resonance origin of the exchange potential. Besides, the short-range impurity scattering cannot be described in the effective mass approximation. ${ }^{38}$ Nevertheless, the estimate of the magnetic component $|J|=1.05 \mathrm{eV}$ is in a good agreement with the corresponding parameter of our theory $\bar{z} V^{2} / E_{\mathrm{CFR}}=1.034 \mathrm{eV}$ used in fitting in Fig. 3. (The value, $W=0.027 \mathrm{eV}$, is irrelevant because of the above-mentioned problem with the effective mass approximation.) One should emphasize, however, that the double-exchange coupling in our model is determined not by this parameter, but by the integral $\Delta E_{\mathrm{FM}}$ (19), and this difference is in fact the benchmark for the discussion of the differences between the indirect $p d$ exchange of Vonsovskii-Zener-type ${ }^{31}$ and the kinematic double exchange. ${ }^{30}$

A simplified picture of the valence band structure in our model (single heavy-hole band with semielliptical density of states) allows us to describe the dependence $T_{C}(x, p)$ using a minimal number of fitting parameters. We have seen that a good quantitative agreement between the theory and experiment is achieved even with this very restricted set of parameters. In a more realistic energy band scheme, e.g., taking the light hole band into account, the restrictive symmetry of the density of states may be removed, and the fitting procedure 
may become more flexible. We intentionally impose such severe restrictions on the model to demonstrate its explanatory capabilities. They may be lifted in future studies.

More accurate treatment of the structure of the valence band is necessary, in particular, for the explanation of transport properties of $p$-( $\mathrm{Ga}, \mathrm{Mn})$ As (anomalous Hall effect and magnetoresistivity). These phenomena are predetermined by the scattering mechanisms and spontaneous magnetization of the carriers, i.e., holes described by the six-band KohnLuttinger Hamiltonian (see Ref. 59 for details). The scattering potential is due to compensating Mn interstitials and As antisite defects, and the anomalous Hall effect occurs because of the spin-orbital interaction in the valence bands. All these phenomena are insensitive to the details of the exchange interaction between substitutional Mn centers. This means that our microscopic theory will give the same picture of magnetotransport as the phenomenological approach, ${ }^{59}$ provided the detailed structure of the valence bands is taken into account. A similar statement can be made about unusual magnetocrystalline anisotropy, which is completely predetermined by the geometry of six-band hole Fermi surface. . $^{15,60}$

Further progress in the theoretical description of ferromagnetism in Mn-doped semiconductors is intimately related with the progress in sample preparation and characterization. At present more or less detailed data on $T_{C}(x, p)$ in $p$-type $(\mathrm{Ga}, \mathrm{Mn})$ As are available, and we have succeeded in a quantitative description of these data within our model (Sec. III). We expect that the same approach is applicable to $p$-type $(\mathrm{Ga}, \mathrm{Mn}) \mathrm{P}$, but the scanty experimental data do not allow us to check this expectation. As for $(\mathrm{Ga}, \mathrm{Mn}) \mathrm{N}$, the accumulation of experimental data is in progress, and the most actual task is to reveal distinctions between $p$ and $n$ type magnetic alloys. Recent experimental data ${ }^{61}$ confirm existence of magnetic order in $n-(\mathrm{Ga}, \mathrm{Mn}) \mathrm{N}$, although the experimental stateof-the-art is far from providing trustworthy $T_{C}(x)$ curves for theoretical fitting. According to our theory, FM order is expected both in $p$ - and $n$-type samples, but the latter case demands a serious modification of the theory (Sec. IV) and a direct extrapolation of the semi-empirical formula (30) is questionable.

Another challenging question is the possibility of ferromagnetism in elemental semiconductors and II-VI compounds doped by $\mathrm{Mn}$ and, maybe, other magnetic ions and respective modification of the theory. One can mention the recent reports about magnetism in Ge doped by Mn (Ref. 62) and $\mathrm{ZnO}$ doped by $\mathrm{Mn}$ and $\mathrm{Fe} .{ }^{63} \mathrm{In}$ the latter case one deals with a wide-gap semiconductor, where magnetic isoelectronic impurities $\mathrm{Mn}^{2+}$ and $\mathrm{Fe}^{2+}$ replace $\mathrm{Zn}$ ions. The theory presented in Sec. IV seems to be applicable in this case. Transition metal ions in $\mathrm{Ge}$ are as a rule interstitial impurities sometimes involved in formation of complex defects, ${ }^{27,28,47}$ so the existing theoretical approaches should be modified for this case. The most important conclusion from our study is that the electronic structure of an isolated magnetic impurity in the host material is the key to understanding its behavior in concentrated magnetic alloys.

\section{ACKNOWLEDGMENTS}

This work was supported by the Flemish Science Foundation (FWO-VI), the Belgian Inter-University Attraction
Poles (IUAP), the "Onderzoeksraad van de Universiteit Antwerpen" (GOA) and the Israeli Physical Society. V.F. and K.K. are grateful to Max-Planck Institute for Complex Systems (Dresden) for partial support and hospitality. V.A.I. is grateful to Grenoble High Magnetic Field Laboratory (Centre National de la Recherche Scientifique and the MaxPlanck Gesellschaft: CNRS-MPI)(Grenoble) for support and hospitality. The authors acknowledge useful and fruitful discussions with J. Cibert, K.W. Edmonds, B. Gallagher, H. Hori, Y.H. Jeong, T. Jungwirth, I. Ya. Korenblit, J. Kudrnovsky, C. Lacroix, A. MacDonald, H. Mariette, and S. Sonoda.

\section{APPENDIX: ENERGY SPECTRUM OF TWO COUPLED IMPURITIES}

The system of four Dyson equations for the $d$-electron Green functions $G_{d i i^{\prime}}^{\sigma}\left(i, i^{\prime}\right)$ has the following form within the Hartree approximation:

$$
\begin{aligned}
G_{d i i^{\prime}}^{\sigma}(\varepsilon)= & g_{d \sigma}(\varepsilon)\left(\delta_{i i^{\prime}}+\sum_{\mathbf{p p}^{\prime}} \sum_{j=1,2} V_{\mathbf{p} d} V_{\mathbf{p}^{\prime} d}^{*} e^{-i \mathbf{p} \cdot \mathbf{R}_{i}}\right. \\
& \left.\times G_{\mathbf{p} \mathbf{p}^{\prime}}^{\sigma} e^{i \mathbf{p}^{\prime} \cdot \mathbf{R}_{j} G_{d j i^{\prime}}^{\sigma}}\right)
\end{aligned}
$$

Here

$$
g_{d \sigma}(\varepsilon) \delta_{i j}=\left(\varepsilon-E_{d}-U n_{i}^{-\sigma}+i \delta \operatorname{sign}(\varepsilon-\mu)\right)^{-1} \delta_{i j}
$$

is the bare single site $d$-electron Green function for the $t_{2} \sigma$ electron centered at the ion $i$, taken in the Hartree approximation. $\mu$ is the chemical potential. The Green function for the band electrons can be found from the Dyson equation (a similar system of equations for two Anderson impurities in metal was analyzed in Ref. 45)

$$
\begin{aligned}
\bar{G}_{\mathbf{p p}^{\prime}}^{\sigma}(\varepsilon)= & G_{\mathbf{p p}^{\prime}}^{\sigma}(\varepsilon)+\sum_{\mathbf{p}^{\prime \prime} \mathbf{p}^{\prime \prime \prime} i j} G_{\mathbf{p p}^{\prime \prime}}^{\sigma}(\varepsilon) V_{\mathbf{p}^{\prime \prime} d} g_{d \sigma}(\varepsilon) V_{\mathbf{p}^{\prime \prime \prime}}^{*} d \\
& \times \bar{G}_{\mathbf{p}^{\prime \prime \prime} \mathbf{p}^{\prime}}^{\sigma}(\varepsilon) e^{i\left(\mathbf{p}^{\prime \prime} \cdot \mathbf{R}_{i}-\mathbf{p}^{\prime \prime \prime} \cdot \mathbf{R}_{j}\right)} .
\end{aligned}
$$

The Green function $G_{\mathbf{p} p^{\prime}}^{\sigma}$ describes the spectrum of the valence band electrons modified by the two-impurity shortrange potential scattering. It satisfies the Dyson equation

$$
G_{\mathbf{p p}^{\prime}}^{\sigma}(\varepsilon)=g_{\mathbf{p}, \sigma}(\varepsilon) \delta_{\mathbf{p p}^{\prime}}+g_{\mathbf{p}, \sigma}(\varepsilon) \sum_{\mathbf{p}^{\prime \prime}} W_{\mathbf{p p}^{\prime \prime}} G_{\mathbf{p}^{\prime \prime} \mathbf{p}^{\prime}}^{\sigma}(\varepsilon),
$$

where

$$
g_{\mathbf{p}, \sigma}(\varepsilon)=\left(\varepsilon-\varepsilon_{\mathbf{p}}+i \delta \operatorname{sign}(\varepsilon-\mu)\right)^{-1} .
$$

All the above equations give linear relations between the Green functions. We may rewrite Eq. (A4) using shorthand notations for the Green function matrices

$$
\mathrm{G}_{b}=\mathrm{A} \cdot \mathrm{g}_{b},
$$

where $g_{b}$ stands for the diagonal matrix (A5). Equations (A1)-(A4) take the form

$$
\mathrm{G}=\mathrm{B} \cdot \mathrm{g}_{0},
$$

where 


$$
g_{0}=\left(\begin{array}{cc}
G_{b} & 0 \\
0 & g_{d}
\end{array}\right)
$$

with $g_{d}$ being a $2 \times 2$ matrix (A2). The explicit expressions for the matrices $A$ and $B$ can be readily found from the Dyson equations (A1) and (A4).

For the further calculations we need determinants of the matrices $A$ and $B$,

$$
\mathrm{Q}(\varepsilon) \equiv \operatorname{det} \mathrm{A}=\mathrm{q}(\varepsilon)^{2}-W^{2} L_{12}(\varepsilon) L_{21}(\varepsilon),
$$

where

$$
\mathrm{q}(\varepsilon)=1-W L_{11}(\varepsilon)
$$

and

$$
L_{i j}=\sum_{\mathbf{p}} g_{p}^{0} e^{i \mathbf{p} \cdot\left(\mathbf{R}_{i}-\mathbf{R}_{j}\right)}
$$

is the lattice Green function. We assume that $L_{11}=L_{22}$. The second determinant is

$$
\mathrm{R} \equiv g_{d}^{-2}(\varepsilon) \operatorname{det} \mathrm{B}=\left[g_{d}^{-1}(\varepsilon)-V^{2} M_{11}^{\sigma}(\varepsilon)\right]^{2}-V^{4} M_{12}^{\sigma}(\varepsilon) M_{21}^{\sigma}(\varepsilon) .
$$

Here

$$
\begin{aligned}
M_{11}^{\sigma} & =\sum_{\mathbf{p} \mathbf{p}^{\prime}} e^{-i\left(\mathbf{p}-\mathbf{p}^{\prime}\right) \cdot \mathbf{R}_{1}} G_{\mathbf{p p}^{\prime}}^{\sigma} \\
& =L_{11}+W Q^{-1}\left[L_{11}^{2} \mathbf{q}+L_{12} L_{21} \mathbf{q}+2 W L_{11} L_{12} L_{21}\right] .
\end{aligned}
$$

$M_{22}^{\sigma}$ is obtained from Eq. (A11) by exchanging indices 1 and 2 , and

$$
\begin{aligned}
M_{12}^{\sigma} & =\sum_{\mathbf{p p}^{\prime}} e^{-i \mathbf{p} \cdot \mathbf{R}_{1}+i \mathbf{p}^{\prime} \cdot \mathbf{R}_{2}} G_{\mathbf{p p}^{\prime}}^{\sigma} \\
& =L_{12}+W Q^{-1}\left[L_{12} L_{11} \mathbf{q}+L_{21} L_{11} \mathbf{q}+W L_{21}^{2} L_{12}\right] .
\end{aligned}
$$

Until now the orbital degeneracy of the impurity $d$ states was neglected and the Hartree approximation was applied. The more general Hubbard I approximation for the $d$-electron Green functions (see, e.g., Ref. 46) can be used for the three-fold degenerate impurity $t_{2 g}$. The algebraic structure of the Dyson equation is still the same as in (A1). Then considering the interaction of two threefold degenerate states of two impurities one gets the $6 \times 6$ matrix

$$
\mathrm{B}^{\prime}{ }_{i \mu, j \mu^{\prime}}=\left(\begin{array}{llllll}
a & 0 & 0 & b & b & b \\
0 & a & 0 & b & b & b \\
0 & 0 & a & b & b & b \\
b & b & b & a & 0 & 0 \\
b & b & b & 0 & a & 0 \\
b & b & b & 0 & 0 & a
\end{array}\right)
$$

where $a=g_{d}^{-1}(\varepsilon)-V^{2} K M_{11}^{\sigma}(\varepsilon), \quad b=V^{2} K M_{12}^{\sigma}(\varepsilon)$, and $K=n_{d^{5}}$ $+n_{d^{4}} \cdot n_{d^{5}}$ is the probability that the impurity $d$-shell is in the nondegenerate $d^{5}$ state, whereas $n_{d^{4}}$ is the probability that the impurity $d$-shell is in one of the three degenerate $d^{4}$ states.
Calculating now the determinant of the matrix (A13) one gets the equation

$$
\begin{aligned}
\mathrm{R} & \equiv a^{-4} \operatorname{det} \mathrm{B}^{\prime} \\
& =\left[g_{d}^{-1}(\varepsilon)-V^{2} K M_{11}^{\sigma}(\varepsilon)\right]^{2}-9 V^{4} K^{2} M_{12}^{\sigma}(\varepsilon) M_{21}^{\sigma}(\varepsilon) .
\end{aligned}
$$

which should be used instead of Eq. (A10).

The occupation numbers $n_{d 5}$ and $n_{d 4}$ for the Hubbard-type states obey a non-Fermi statistics, whose specific form in the case considered here is

$$
n_{d 5}=\frac{f\left(E_{\mathrm{CFR}}-\mu\right)}{3-2 f\left(E_{\mathrm{CFR}}-\mu\right)}, \quad n_{d 4}=\frac{1-f\left(E_{\mathrm{CFR}}-\mu\right)}{3-2 f\left(E_{\mathrm{CFR}}-\mu\right)} .
$$

If the chemical potential lies below the impurity level $E_{\mathrm{CFR}}$ of the fifth electron in the $d$-shell, then the Fermi distribution $f\left(E_{\mathrm{CFR}}-\mu\right)$ is zero at low temperatures and $n_{d 5}=0, n_{d 4}$ $=1 / 3$, meaning that $K=1 / 3$. If $E_{\mathrm{CFR}}<\mu$, then $n_{d 5}=1, n_{d 4}$ $=0$, and $K=1$.

Using the general property of the Green functions

$$
\operatorname{Tr} \mathrm{G}(\varepsilon)=\frac{d}{d \varepsilon} \ln \operatorname{det} \mathrm{G}(\varepsilon)
$$

and Eqs. (A8), (A10), and (A16), Eq. (5) may be rewritten in the form

$$
\begin{aligned}
\Delta E & =\operatorname{Im} \int_{-\infty}^{\infty} \frac{d \varepsilon}{2 \pi} \varepsilon \frac{d}{d \varepsilon}\left[\ln \mathrm{R}^{\sigma}(\varepsilon)+\ln \mathrm{Q}^{\sigma}(\varepsilon)\right] \\
& =-\operatorname{Im} \int_{-\infty}^{\infty} \frac{d \varepsilon}{2 \pi}\left[\ln \mathrm{R}^{\sigma}(\varepsilon)+\ln \mathrm{Q}^{\sigma}(\varepsilon)\right]
\end{aligned}
$$

Since the functions $\mathrm{R}$ and $\mathrm{Q}$ depend on the combination $\varepsilon$ $-i \operatorname{sign}(\varepsilon-\mu)$, the integration contour in (A17) can be deformed in such a way as to embrace the cut from the band states and all the poles due to the localized levels with the energies below the chemical potential $\mu$, i.e., occupied states. Then Eq. (A17) transforms into (6).

Next we consider a property of Eq. (A17), which will simplify the calculation of the energy and provides a better intuition to the results. Our model includes all the levels belonging to the valence band with the addition of the impurity $d$-levels, which interact with the band levels. Let us assume that at zero temperature the chemical potential lies higher than all these levels, meaning that they are all occupied. Then the total energy of the system is $E_{\text {tot }}=\operatorname{Tr} \hat{H}$. The trace of the operator $\hat{H}$ does not depend on the off-diagonal hybridization matrix elements.

The potential scattering, $W_{\mathbf{p p}^{\prime}}$, has diagonal elements and may influence the value of $E_{\text {tot }}$. However, we are interested here only in the indirect exchange between the impurities. The latter can be found if we consider the energy $\Delta E\left(R_{i j}\right)$ and subtract from it the energy corresponding to two noninteracting impurities, 


$$
\begin{aligned}
\Delta E_{\mathrm{ex}}= & -\frac{1}{\pi} \operatorname{Im} \int_{\varepsilon_{h b}}^{\mu} d \varepsilon\left[\ln \frac{\mathrm{R}^{\sigma}(\varepsilon)}{\mathrm{R}_{0}^{\sigma}(\varepsilon)}+\ln \frac{\mathrm{Q}^{\sigma}(\varepsilon)}{\mathrm{Q}_{0}^{\sigma}(\varepsilon)}\right] \\
& +\Delta E_{\mathrm{loc}}(\varepsilon<\mu),
\end{aligned}
$$

where $\mathrm{Q}_{0}^{\sigma}(\varepsilon)$ and $\mathrm{R}_{0}^{\sigma}(\varepsilon)$ are obtained from (A8) and (A10) under the assumption that $L_{12}=L_{21}=0 . \Delta E_{\mathrm{loc}}(\varepsilon<\mu)$ is the corresponding change of the energies of the occupied localized levels. The sum (A18) over all occupied states is equal to the same sum over all empty states, but with the opposite sign. Hence,

$\Delta E_{\mathrm{ex}}=\frac{1}{\pi} \operatorname{Im} \int_{\mu}^{\varepsilon_{h t}} d \varepsilon\left[\ln \frac{\mathrm{R}^{\sigma}(\varepsilon)}{\mathrm{R}_{0}^{\sigma}(\varepsilon)}+\ln \frac{\mathrm{Q}^{\sigma}(\varepsilon)}{\mathrm{Q}_{0}^{\sigma}(\varepsilon)}\right]-\Delta E_{\mathrm{loc}}(\varepsilon>\mu)$.

Here $\Delta E_{\text {loc }}(\varepsilon>\mu)$ includes the empty localized levels (if any) lying above the chemical potential. These levels, which are defined as zeros of the determinant R (A10), appear due to combined action of potential $(W)$ and resonance $(V)$ scattering mechanisms.

To find the contribution of localized states to the magnetic energy one should simply calculate the level positions modified by the effective inter-impurity exchange via empty states and their occupation. We consider here two limiting cases. First, we estimate the contribution of CFR levels, if they happen to lie within the forbidden energy gap. For this sake we expand the function $\mathrm{R}(\varepsilon)$ close to the energy $E_{\mathrm{CFR} \sigma}^{0}$ of the isolated CFR level determined by the equation

$$
E_{\mathrm{CFR}}^{0}=E_{d}+K V^{2} P_{11}\left(E_{\mathrm{CFR}}^{0}\right),
$$

which describes the TM $d$-levels renormalized by their hybridization with the $h h$ band. Indirect interimpurity interaction results in splitting of two-impurity states and a shift of localized states relative to the $h h$ band. These levels lie in the discrete part of the spectrum, where the imaginary part of the Green function (9) $\Gamma_{i j}=0$. Neglecting potential scattering, we obtain the equation

$$
\begin{aligned}
\mathrm{R}= & {\left[\varepsilon-E_{d}-K V^{2} P_{11}^{\sigma}(\varepsilon)\right]\left[\varepsilon-E_{d}-K V^{2} P_{22}^{\sigma}(\varepsilon)\right] } \\
& -9 K^{2} V^{4} P_{12}^{\sigma}(\varepsilon) P_{21}^{\sigma}(\varepsilon)=0
\end{aligned}
$$

for the two-impurity poles in the energy gap. The solution of
Eq. (A21) is looked for in the form $E_{\mathrm{CFR}}=E_{\mathrm{CFR}}^{0}+\delta E_{\mathrm{CFR}}$. Then we expand the function $\mathrm{R}\left(E_{\mathrm{CFR}}^{0}+\delta E_{\mathrm{CFR}}\right)$ up to the second order terms with respect to $\delta E_{\mathrm{CFR}}$ and arrive at Eq. (11).

Second, we consider the case when the DBH levels lie in the forbidden energy gap. In this case the potential scattering $W$ is the leading cause of the creation of the deep level. The energy of an isolated DBH level corresponds to a zero of the function

$$
\mathrm{q}\left(E_{\mathrm{DBH}}^{0}\right)=1-W P_{11}\left(E_{\mathrm{DBH}}^{0}\right)=0 .
$$

Then we look for zeros of the function $R(\varepsilon)$ at the energy $E_{\mathrm{DBH}}=E_{\mathrm{DBH}}^{0}+\delta E_{\mathrm{DBH}}$. Accounting for the fact that both functions $q$ and $Q$ are small in the vicinity of the energy $E_{\mathrm{DBH}}^{0}$ the equation $R=0$ can be approximately rewritten in the form

$$
\begin{gathered}
\left\{\mathrm{q}^{2}-W^{2} P_{12}^{2}-\frac{K V^{2}}{\Delta E} W\left[P_{11}^{2} \mathrm{q}+P_{12}^{2} \mathrm{q}+2 W P_{11} P_{12}^{2}\right]\right\}^{2} \\
=\frac{9 K^{2} V^{4}}{\Delta E^{2}}\left\{P_{12}+W\left[2 P_{12} P_{11} \mathrm{q}+W P_{12}^{2}\right]\right\}^{2}
\end{gathered}
$$

with $\Delta E=E_{\mathrm{DBH}}^{0}-E_{d}-V^{2} P_{11}$. All the functions $P_{i j}$ are now calculated at $\varepsilon=E_{\mathrm{DBH}}^{0}$. We first neglect the r.h.s. term in Eq. (A22) and solve the quadratic equation

$$
\mathrm{q}^{2}-\frac{K V^{2}}{\Delta E} W\left[P_{11}^{2}+P_{12}^{2}\right] \mathrm{q}-W^{2} P_{12}^{2}-\frac{2 K V^{2} W^{2}}{\Delta E} P_{11} P_{12}^{2}=0 .
$$

From here we obtain Eq. (16) for the energy shifts due to interaction between the two degenerate DBH levels. When both these levels are empty we obtain a contribution to the kinematic exchange by summing these two energies, extracting from them the part due to the hybridization with the impurity $d$-states, and changing the sign in the hole representation,

$$
\Delta E_{\mathrm{DBH}, \mathrm{ex}}=\frac{K V^{2} P_{12}^{2}}{\Delta E P_{11}^{\prime}}
$$

Accounting for the r.h.s. of Eq. (A22) will result in higher order corrections, which can be neglected.
*Electronic address: fleurov@post.tau.ac.il

${ }^{1}$ M. Ilegems, R. Dingle, and L.W. Rupp, Jr., J. Appl. Phys. 46, 3059 (1975); D.G. Andrianov, V.V. Karataev, G.V. Lazarev, Yu.B. Muravlev, and A.S. Savel'ev, Sov. Phys. Semicond. 11, 738 (1977); T. Hayashi, Y. Hashimoto, S. Katsumoto, and Y. Iye, Appl. Phys. Lett. 78, 1691 (2001).

${ }^{2}$ F. Matsukura, H. Ohno, A. Shen, and Y. Sugawara, Phys. Rev. B 57, R2037 (1998).

${ }^{3}$ J. M. Kikkawa and A. D. Awschalom, Nature (London) 397, 139 (1998).

${ }^{4}$ H. Ohno, H. Munekata, T. Penney, S. von Molnár, and L. L.
Chang, Phys. Rev. Lett. 68, 2664 (1992).

${ }^{5}$ H. Ohno, J. Magn. Magn. Mater. 200, 110 (1999); Science 281, 951 (1998).

${ }^{6}$ K. W. Edmonds, K. Y. Wang, R. P. Campion, A. C. Neumann, N. R. S. Farley, B. L. Gallagher, and C. T. Foxon, Appl. Phys. Lett. 81, 4991 (2002).

${ }^{7}$ I. Kuryliszyn, T. Wojtowicz, X. Liu, J. K. Furdyna, W. Dobrowolski, J.-M. Broto, M. Goiran, O. Portugal, H. Rakoto, and B. I. Raquet, Acta Phys. Pol. A 102, 659 (2002).

${ }^{8}$ K. C. Ku, S. J. Potashnik, R. F. Wang, S. H. Chun, P. Schiffer, and N. Samarth, M. J. Seong, A. Mascarenhas, E. Johnston- 
Halperin, R. C. Myers, A. C. Gossard, and D. D. Awschalom, Appl. Phys. Lett. 82, 2302 (2003); K.W. Edmonds, P. Boguslawski, K.Y. Wang, R.P. Campion, N.R.S. Farley, B.L. Gallagher, C.T. Foxon, M. Sawicki, T. Dietl, M.B. Nardelli, and J. Bernholc, cond-mat/0307140 (unpublished).

${ }^{9}$ D. Chiba, K. Takamura, F. Matsulura, and H. Ohno, Appl. Phys. Lett. 82, 3020 (2003).

${ }^{10}$ B. Gallagher, CECAM Workshop: "Diluted Magnetic Semiconductors," June 12-14, 2003, Lyon.

${ }^{11}$ S. Sonoda, S. Shimizu, T. Sasaki, Y. Yamamoto, and H. Hori, J. Appl. Phys. 91, 7911 (2002).

${ }^{12}$ M. L. Reed, N. A. El-Masry, H. H. Stadelmaier, M. K. Ritums, M. J. Reed, C. A. Parker, J. C. Roberts, and S. M. Bedair, Appl. Phys. Lett. 79, 3473 (2001); N. Theodoropolou, A. F. Hebard, M. E. Overberg, S. N. G. Chu, and R. G. Wilson, ibid. 78, 3475 (2001); M. E. Overberg, C. R. Abernathy, S. J. Pearton, N. A. Theodoropoulou, K. T. McCarthy, and A. F. Hebard, ibid. 79, 1312 (2001).

${ }^{13}$ H. Ohno, A. Shen, F. Matsukara, O. Oiwa, A. Endo, S. Katsumoto, and Y. Iye, Appl. Phys. Lett. 69, 363 (1996).

${ }^{14}$ T. Dietl, H. Ohno, F. Matsukura, J. Cibert, and D. Ferrand, Science 287, 139 (1998).

${ }^{15}$ T. Dietl, H. Ohno, and F. Matsukura, Phys. Rev. B 63, 195205 (2001).

${ }^{16}$ T. Dietl, F. Matsukura, and H. Ohno, Phys. Rev. B 66, 033203 (2002).

${ }^{17}$ M. Berciu and R. N. Bhatt, Phys. Rev. Lett. 87, 107203 (2001).

${ }^{18}$ Yu. G. Semenov and S.M. Ryabchenko, Low Temp. Phys. 26, 886 (2000) [Fiz. Nizk. Temp. 26, 1197 (2000)].

${ }^{19}$ V. K. Dugaev, V.I. Litvinov, J. Barnaś, and M. Vieira, Phys. Rev. B 67, 033201 (2003).

${ }^{20}$ J. Inoue, S. Nonoyama, and H. Itoh, Phys. Rev. Lett. 85, 4610 (2000); Physica E (Amsterdam) 10, 170 (2001).

${ }^{21}$ A. Singh, A. Datta, S.K. Das, and V.A. Singh, Phys. Rev. B 68, 235208 (2003).

${ }^{22}$ S. Sanvito, P. Ordejón, and N.A. Hill, Phys. Rev. B 63, 165206 (2001).

${ }^{23}$ K. Sato and H. Katayama-Yoshida, Jpn. J. Appl. Phys., Part 140 , L485 (2001); P. Mahadevan and A. Zunger, "Electronic structure and ferromagnetism of $3 d$ transition metal impurities in GaAs" (preprint); L. Kronik, M. Jain, and J. R. Chelikowsky, Phys. Rev. B 66, 041203(R) (2002); M. van Schilfgaarde and O. N. Mryasov, ibid. 63, 233205 (2001); E. Kulatov, H. Nakayama, H. Mariette, H. Ohta, and Y. A. Uspenskii, ibid. 66, 045203 (2002).

${ }^{24}$ K. Sato, P. H. Dederichs, and H. Katayama-Yoshida, Europhys. Lett. 61, 403 (2003); K. Sato, P. H. Dederichs, H. KatayamaYoshida, and J. Kudrnovsky, Physica B 340-342, 863 (2003).

${ }^{25}$ V. N. Fleurov and K. A. Kikoin, J. Phys. C 9, 1673 (1976).

${ }^{26}$ F. D. M. Haldane and P. W. Anderson, Phys. Rev. B 13, 2553 (1976).

${ }^{27}$ K. A. Kikoin and V. N. Fleurov, Transition Metal Impurities in Semiconductors (World Scientific, Singapore, 1994).

${ }^{28}$ A. Zunger, in Solid State Physics, edited by H. Ehrenreich and D. Turnbull (Academic, Orlando, 1986), Vol. 39, p. 276.

${ }^{29}$ P. M. Krstajić, V. A. Ivanov, F. M. Peeters, V. Fleurov, and K. Kikoin, Europhys. Lett. 61, 235 (2003).

${ }^{30}$ C. Zener, Phys. Rev. 82, 403 (1951).

${ }^{31}$ S. V. Vonsovskii, Zh. Eksp. Teor. Fiz. 16, 981 (1946); C. Zener, Phys. Rev. 81, 440 (1951).
${ }^{32}$ P. W. Anderson, Phys. Rev. 124, 41 (1961).

${ }^{33}$ S. Alexander and P. W. Anderson, Phys. Rev. 133, A1594 (1964).

${ }^{34}$ K. A. Kikoin and V. N. Fleurov, J. Phys. C 10, 4295 (1977).

${ }^{35}$ L. A. Hemstreet, Phys. Rev. B 22, 4590 (1980).

${ }^{36}$ G. Picoli, A. Chomette, and M. Lannoo, Phys. Rev. B 30, 7138 (1984).

${ }^{37}$ V. A. Singh and A. Zunger, Phys. Rev. B 31, 3729 (1985).

${ }^{38}$ V. N. Fleurov and K. A. Kikoin, J. Phys. C 19, 887 (1986).

${ }^{39}$ K. A. Kikoin and V. N. Fleurov, J. Phys. C 17, 2357 (1984).

${ }^{40}$ J. Schneider, U. Kaufmann, W. Wilkening, M. Baeumler, and F. Köhl, Phys. Rev. Lett. 59, 240 (1987).

${ }^{41}$ M. Linnarsson, E. Janzen, B. Monemar, M. Kleverman, and A. Thilderkvist, Phys. Rev. B 55, 6938 (1997).

${ }^{42}$ J. Szczytko, A. Twardowski, K. Swiatek, M. Palczewska, M. Tanaka, T. Hayashi, and K. Ando, Phys. Rev. B 60, 8304 (1999).

${ }^{43}$ Y. Nagai, T. Kunimoto, K. Nagasaka, H. Nojiri, M. Motokawa, F. Matsukura, T. Dietl, and H. Ohno, Jpn. J. Appl. Phys., Part 140 , 6231 (2001).

${ }^{44}$ T. Graf, M. Gjukic, L. Görgens, O. Ambacher, M. S. Brandt, and M. Stutzmann, Appl. Phys. Lett. 81, 5159 (2002).

${ }^{45}$ B. Caroli, J. Phys. Chem. Solids 28, 1427 (1967).

${ }^{46}$ K. A. Kikoin and V. N. Fleurov,Zh. Eksp. Teor. Fiz. 77, 1062 (1979) [Sov. Phys. JETP 50, 535 (1979)].

${ }^{47}$ Data in Science and Technology-Semiconductors: Group IV Elements and III-V Compounds, edited by O. Madelung (SpringerVerlag, Berlin, 1991).

${ }^{48}$ K. W. Edmonds, K. Y. Wang, R. P. Campion, A. C. Neumann, C. T. Foxon, B. L. Gallagher, and P. C. Main, Appl. Phys. Lett. 81, 3010 (2002).

${ }^{49}$ It is worth mentioning here that unlike magnetically doped metals (Ref. 45) we cannot represent the effective interimpurity coupling in the asymptotic form $\Delta E_{\mathrm{FM}}\left(R_{i j}\right) \sim \cos \left(2 k_{F} R_{i j}\right.$ $+\phi) /\left(k_{F} R_{i j}\right)^{3}$ because typically $k_{F}^{-1} \sim R_{i j}$ in doped semiconductors.

${ }^{50}$ H. Asklund, L. Ilver, J. Kanski, J. Sadowski, and R. Mathieu, Phys. Rev. B 66, 115319 (2002).

${ }^{51}$ K. M. Yu, W. Walukiewicz, T. Wojtowicz, W. L. Lim, X. Liu, Y. Sasaki, M. Dobrowolska, and J. K. Furdyna, Appl. Phys. Lett. 81, 5 (2002)

${ }^{52}$ N. Theodoropoulou, A. F. Hebard, M. E. Overberg, C. R. Abernathy, S. J. Pearton, S. N. G. Chu, and R. G. Wilson, Phys. Rev. Lett. 89, 107203 (2002).

${ }^{53}$ V. Barzykin, arXiv: cond-mat/0311114 (unpublished).

${ }^{54}$ I. Ya. Korenblit and E. F. Shender, Zh. Eksp. Teor. Fiz. 62, 1949 (1972) [Sov. Phys. JETP 35, 1017 (1972)]; S. L. Ginzburg, I. Ya. Korenblit, and I. Y. Shender, Zh. Eksp. Teor. Fiz. 64, 2255 (1973) [Sov. Phys. JETP 37, 1141 (1973)]; I. Ya. Korenblit and E.F. Shender, Usp. Fiz. Nauk 126, 233 (1978) [Sov. Phys. Usp. 21, 832 (1978)].

${ }^{55}$ I. Ya. Korenblit, E. F. Shender, and B. I. Shklovskii, Phys. Lett. 46A, 275 (1973).

${ }^{56}$ P. W. Anderson, Phys. Rev. 79, 350 (1950); J. B. Goodenough, Magnetism and Chemical Bond (Interscience, New York, 1963).

${ }^{57}$ T. Jungwirth, W. A. Atkinson, B. H. Lee, and A. H. MacDonald, Phys. Rev. B 59, 9818 (1999).

${ }^{58}$ A.A. Abrikosov and L.P. Gorkov, Zh. Eksp. Teor. Fiz. 43, 2230 (1962) [Sov. Phys. JETP 16, 1575 (1963)].

${ }^{59}$ T. Jungwirth, Q. Niu, and A.H. MacDonald, Phys. Rev. Lett. 88, 207208 (2002); T. Jungwirth, M. Abolfath, J. Sinova, J. Kučera, 
and A. MacDonald, Appl. Phys. Lett. 81, 4029 (2002); T. Jungwirth, J. Sinova, K.Y. Wang, K.W. Edmonds, R.P. Campion, B.J. Gallacher, C.T. Foxon, Q. Niu, and A. MacDonald, arXiv: condmat/0302060 (unpublished).

${ }^{60}$ M. Abolfath, T. Jungwirth, J. Brum, and A.H. MacDonald, Phys. Rev. B 63, 054418 (2001).

${ }^{61}$ P.P. Chen, H. Makino, J.J. Kim, and T. Chao, J. Cryst. Growth 251, 331 (2003); R. Giraud, S. Kuroda, S. Marcet, E. BelletAmarlic, X. Biquard, B. Barbara, D. Fruchart, J. Cibert, and H. Mariette, Europhys. Lett. (unpublished).

${ }^{62}$ S. Cho, S. Choi, S.-C. Hong, Y. Kim, J. B. Ketterson, B-J. Kim,
Y. C. Kim, and J-H. Jung, Phys. Rev. B 66, 033303 (2002); A. Stroppa, S. Picozzi, A. Continenza, and A. J. Freeman, ibid. 68, 155203 (2003); Y. D. Park, A. T. Hanbicki, S. C. Erwin, C. S. Hellberg, J. M. Sullivan, J. E. Mattson, T. F. Ambrose, and A. Wilson, Science 295, 651 (2002); F. Tsui, L. He, L. Ma, A. Tkachuk, Y. S. Chu, K. Nakajima, and T. Chikyow, Phys. Rev. Lett. 91, 177203 (2003).

${ }^{63}$ K. Ueda, H. Tabata, and T. Kawai, Appl. Phys. Lett. 79, 988 (2001); D. P. Norton, S. J. Pearton, A. F. Hebard, N. Theodoropoulou, L. A. Boatner, and R. G. Wilson, ibid. 82, 239 (2003). 